\title{
HOPF STRUCTURES ON THE MULTIPLIHEDRA
}

\author{
STEFAN FORCEY, AARON LAUVE, AND FRANK SOTTILE
}

\begin{abstract}
We investigate algebraic structures that can be placed on vertices of the multiplihedra, a family of polytopes originating in the study of higher categories and homotopy theory. Most compelling among these are two distinct structures of a Hopf module over the Loday-Ronco Hopf algebra.
\end{abstract}

\section{INTRODUCTION}

The permutahedra $\mathfrak{S}$. form a family of highly symmetric polytopes that have been of interest since their introduction by Schoute in 1911 [23]. The associahedra $\mathcal{Y}$. are another family of polytopes that were introduced by Stasheff as cell complexes in 1963 [25], and with the permutahedra were studied from the perspective of monoidal categories and $H$-spaces [17] in the 1960s. Only later were associahedra shown to be polytopes $[11,13,18]$. Interest in these objects was heightened in the 1990s, when Hopf algebra structures were placed on them in work of Malvenuto, Reutenauer, Loday, Ronco, Chapoton, and others [6, 14, 16]. More recently, the associahedra were shown to arise in Lie theory through work of Fomin and Zelevinsky on cluster algebras [7].

We investigate Hopf structures on another family of polyhedra, the multiplihedra, M. . Stasheff introduced them in the context of maps preserving higher homotopy associativity [26] and described their 1-skeleta. Boardman and Vogt [5], and then Iwase and Mimura [12] described the multiplihedra as cell complexes, and only recently were they shown to be convex polytopes [8]. These three families of polytopes are closely related. For each integer $n \geq 1$, the permutahedron $\mathfrak{S}_{n}$, multiplihedron $\mathcal{M}_{n}$, and associahedron $\mathcal{Y}_{n}$ are polytopes of dimension $n-1$ with natural cellular surjections $\mathfrak{S}_{n} \rightarrow \mathcal{M}_{n} \rightarrow \mathcal{Y}_{n}$, which we illustrate when $n=4$.
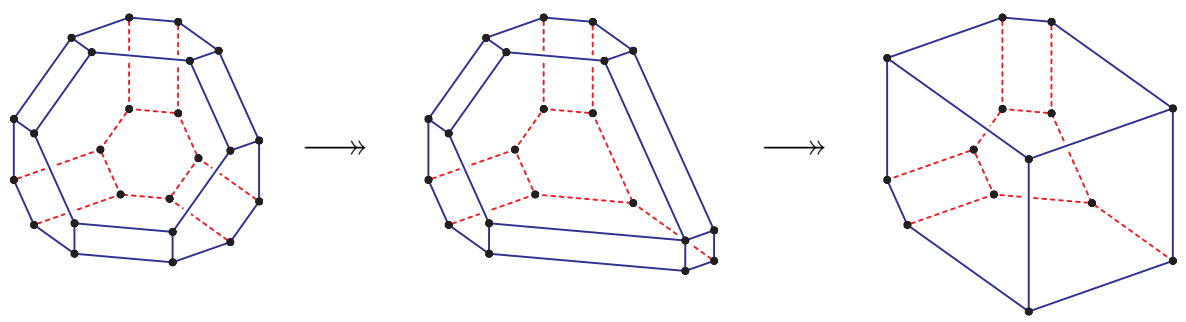

The faces of these polytopes are represented by different flavors of planar trees; permutahedra by ordered trees (set compositions), multiplihedra by bi-leveled trees (Section 2.1), and associahedra by planar trees. The maps between them forget

Key words and phrases. multiplihedron, permutations, permutahedron, associahedron, binary trees, Hopf algebras.

Sottile supported by NSF grant DMS-0701050. 
the additional structure on the trees. These maps induce surjective maps of graded vector spaces spanned by the vertices, which are binary trees. The span $\mathfrak{5} S y m$ of ordered trees forms the Malvenuto-Reutenauer Hopf algebra [16] and the span $\mathcal{Y}$ Sym of planar binary trees forms the Loday-Ronco Hopf algebra [14]. The algebraic structures of multiplication and comultiplication on $\mathfrak{S} S y m$ and $\mathcal{Y}$ Sym are described in terms of geometric operations on trees and the composed surjection $\tau: \mathfrak{S}$. $\rightarrow \mathcal{Y}$. gives a surjective morphism $\tau: \mathfrak{S} S y m \rightarrow \mathcal{Y}$ Sym of Hopf algebras.

We define $\mathcal{M}$ Sym to be the vector space spanned by the vertices of all multiplihedra. The factorization of $\tau$ induced by the maps of polytopes, $\mathfrak{S} S y m \rightarrow$ $\mathcal{M}$ Sym $\rightarrow \mathcal{Y}$ Sym, does not endow $\mathcal{M}$ Sym with the structure of a Hopf algebra. Nevertheless, some algebraic structure does survive the factorization. We show in Section 3 that $\mathcal{M S y m}$ is an algebra, which is simultaneously a $\mathfrak{S} S y m$-module and a $\mathcal{Y}$ Sym-Hopf module algebra, and the maps preserve these structures.

We perform a change of basis in $\mathcal{M}$ Sym using Möbius inversion that illuminates its comodule structure. Such changes of basis helped to understand the coalgebra structure of SSym [1] and of $\mathcal{Y}$ Sym [2]. Section 4 discusses a second $\mathcal{Y}$ Sym Hopf module structure that may be placed on the positive part $\mathcal{M} S y m_{+}$of $\mathcal{M S y m}$. This structure also arises from polytope maps between $\mathfrak{S}$. and $\mathcal{Y}_{\bullet}$, but not directly from the algebra structure of $\mathfrak{S} S y m$. Möbius inversion again reveals an explicit basis of $\mathcal{Y}$ Sym coinvariants in this alternate setting.

\section{Basic Combinatorial Data}

The structures of the Malvenuto-Reutenauer and Loday-Ronco algebras are related to the weak order on ordered trees and the Tamari order on planar trees. There are natural maps between the weak and Tamari orders which induce a morphism of Hopf algebras. We first recall these partial orders and then the basic structure of these Hopf algebras. In Section 1.3 we establish a formula involving the Möbius functions of two posets related by an interval retract. This is a strictly weaker notion than that of a Galois correspondence, which was used to study the structure of the Loday-Ronco Hopf algebra.

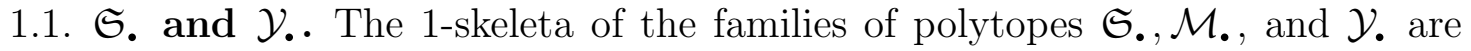
Hasse diagrams of posets whose structures are intertwined with the algebra structures we study. We use the same notation for a polytope and its poset of vertices. Similarly, we use the same notation for a cellular surjection of polytopes and the poset map formed by restricting that surjection to vertices.

For the permutahedron $\mathfrak{S}_{n}$, the corresponding poset is the (left) weak order, which we describe in terms of permutations. A cover in the weak order has the form $w \lessdot(k, k+1) w$, where $k$ preceeds $k+1$ among the values of $w$. Figure 1 displays the weak order on $\mathfrak{S}_{4}$. We let $\mathfrak{S}_{0}=\{\emptyset\}$, where $\emptyset$ is the empty permutation of $\emptyset$.

Let $\mathcal{Y}_{n}$ be the set of rooted, planar binary trees with $n$ nodes. The cover relations in the Tamari order on $\mathcal{Y}_{n}$ are obtained by moving a child node directly above a given node from the left to the right branch above the given node. Thus

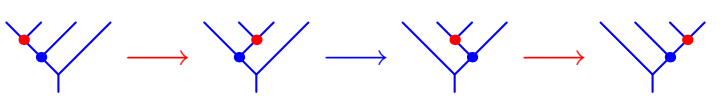


is an increasing chain in $\mathcal{Y}_{3}$ (the moving vertices are marked with dots). Figure 1 shows the Tamari order on $\mathcal{Y}_{4}$.
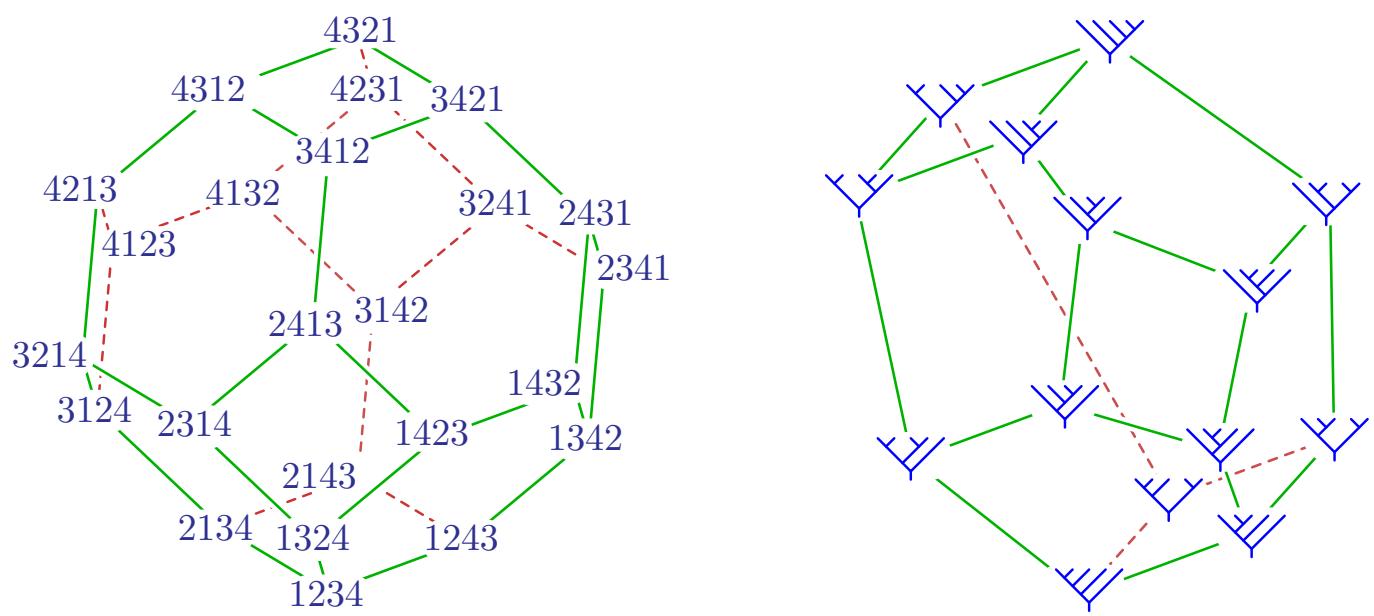

FiguRE 1. Weak order on $\mathfrak{S}_{4}$ and Tamari order on $\mathcal{Y}_{4}$

The unique tree in $\mathcal{Y}_{1}$ is $Y$. Given trees $t_{\ell}$ and $t_{r}$, form the tree $t_{\ell} \vee t_{r}$ by grafting the root of $t_{\ell}$ (respectively of $t_{r}$ ) to the left (respectively right) leaf of $Y$. Form the tree $t_{\ell} \backslash t_{r}$ by grafting the root of $t_{r}$ to the rightmost leaf of $t_{\ell}$. For example,
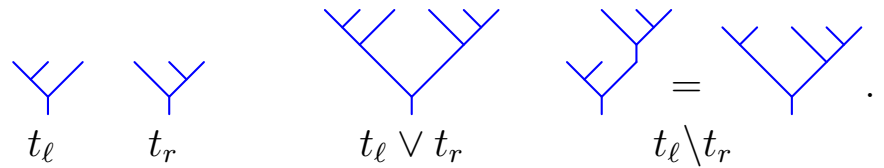

Decompositions $t=t_{1} \backslash t_{2}$ correspond to pruning $t$ along the right branches from the root. A tree $t$ is indecomposable if it has no nontrivial decomposition $t=t_{1} \backslash t_{2}$ with $t_{1}, t_{2} \neq 1$. Equivalently, if the root node is the rightmost node of $t$. Any tree $t$ is uniquely decomposed $t=t_{1} \backslash \cdots \backslash t_{m}$ into indecomposable trees $t_{1}, \ldots, t_{m}$.

We define a poset map $\tau: \mathfrak{S}_{n} \rightarrow \mathcal{Y}_{n}$. First, given distinct integers $a_{1}, \ldots, a_{k}$, let $\bar{a} \in \mathfrak{S}_{k}$ be the unique permutation such that $\bar{a}(i)<\bar{a}(j)$ if and only if $a_{i}<a_{j}$. Thus $\overline{4726}=2413$. Since $\mathfrak{S}_{0}, \mathcal{Y}_{0}, \mathfrak{S}_{1}$, and $\mathcal{Y}_{1}$ are singletons, we must have

$$
\begin{aligned}
& \tau: \mathfrak{S}_{0} \longrightarrow \mathcal{Y}_{0} \text { with } \tau: \emptyset \longmapsto \mid \text {, and } \\
& \tau: \mathfrak{S}_{1} \longrightarrow \mathcal{Y}_{1} \text { with } \tau: 1 \longmapsto Y \text {. }
\end{aligned}
$$

Let $n>0$ and assume that $\tau$ has been defined on $\mathfrak{S}_{k}$ for $k<n$. For $w \in \mathfrak{S}_{n}$ suppose that $w(j)=n$, and define

$$
\tau(w):=\tau(\overline{w(1), \ldots, w(j-1)}) \vee \tau(\overline{w(j+1), \ldots, w(n}) .
$$

For example,

$$
\begin{gathered}
\tau(12)=Y \vee|=Y, \tau(21)=| \vee Y=Y \text {, and } \\
\tau(3421)=\tau(\overline{3}) \vee \tau(\overline{21})=\tau(1) \vee \tau(21)=Y \vee Y=Y
\end{gathered}
$$


Loday and Ronco [15] show that the fibers $\tau^{-1}(t)$ of $\tau$ are intervals in the weak order. This gives two canonical sections of $\tau$. For $t \in \mathcal{Y}_{n}$,

$$
\min (t):=\min \{w \mid \tau(w)=t\} \quad \text { and } \max (t):=\max \{w \mid \tau(w)=t\},
$$

the minimum and maximum in the weak order. Equivalently, $\min (t)$ is the unique 231-avoiding permutation in $\tau^{-1}(t)$ and $\max (t)$ is the unique 132-avoiding permutation. These maps are order-preserving.

The 1-skeleta of $\mathfrak{S}_{n}$ and $\mathcal{Y}_{n}$ form the Hasse diagrams of the weak and Tamari orders, respectively. Since $\tau$ is an order-preserving surjection, it induces a cellular map between the 1-skeleta of these polytopes. Tonks [27] extended $\tau$ to the faces of $\mathfrak{S}_{n}$, giving a cellular surjection.

The nodes and internal edges of a tree are the Hasse diagram of a poset with the root node maximal. Labeling the nodes (equivalently, the gaps between the leaves) of $\tau(w)$ with the values of the permutation $w$ gives a linear extension of the node poset of $\tau(w)$, and all linear extensions of a tree $t$ arise in this way for a unique permutation in $\tau^{-1}(t)$. Such a linear extension $w$ of a tree is an ordered tree and $\tau(w)$ is the corresponding unordered tree. In this way, $\mathfrak{S}_{n}$ is identified with the set of ordered trees with $n$ nodes. Here are some ordered trees,

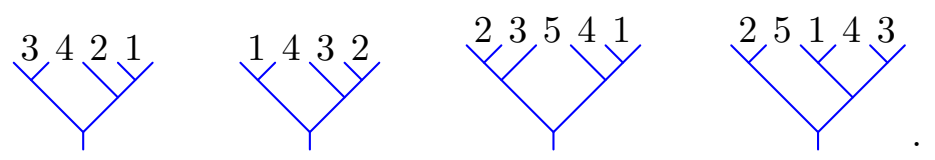

Given ordered trees $u, v$, form the ordered tree $u \backslash v$ by grafting the root of $v$ to the rightmost leaf of $u$, where the nodes of $u$ are greater than the nodes of $v$, but the relative orders within $u$ and $v$ are maintained. Thus we may decompose an ordered tree $w=u \backslash v$ whenever $\tau(w)=r \backslash s$ with $\tau(u)=r, \tau(v)=s$, and the nodes of $r$ in $w$ precede the nodes of $s$ in $w$. An ordered tree $w$ is indecomposable if it has no nontrivial such decompositions. Here are ordered trees $u, v$ and $u \backslash v$,

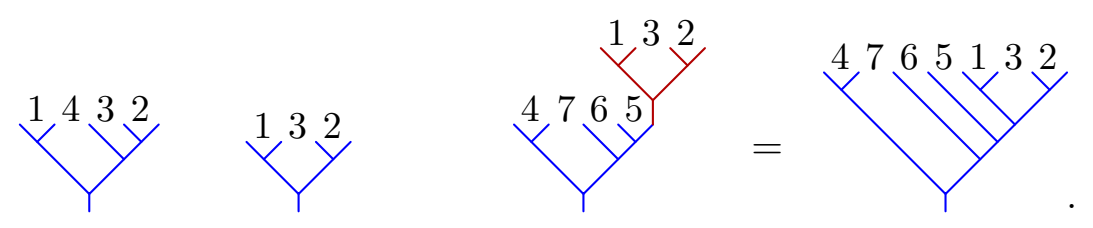

We may split an ordered tree $w$ along a leaf to obtain either an ordered forest (where the nodes in the forest are totally ordered) or a pair of ordered trees,

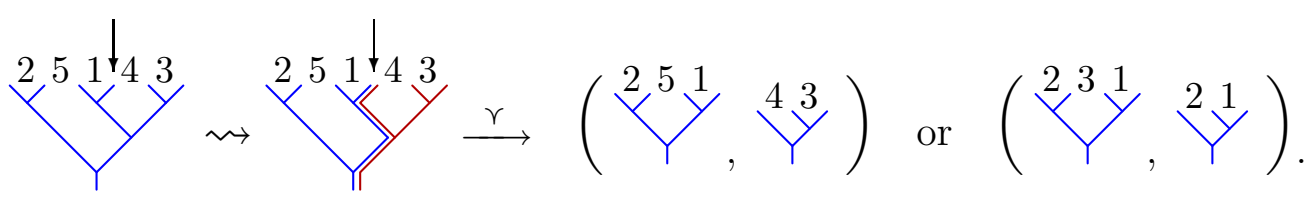

Write $w \stackrel{\curlyvee}{\rightarrow}\left(w_{0}, w_{1}\right)$ to indicate that the ordered forest $\left(w_{0}, w_{1}\right)$ (or pair of ordered trees) is obtained by splitting $w$ along some leaf. (Context will determine how to interpret the result.) More generally, we may split an ordered tree $w$ along a multiset of $m \geq 0$ of its leaves to obtain an ordered forest, or tuple of ordered 
trees, written $w \stackrel{r}{\rightarrow}\left(w_{0}, \ldots, w_{m}\right)$. For example,

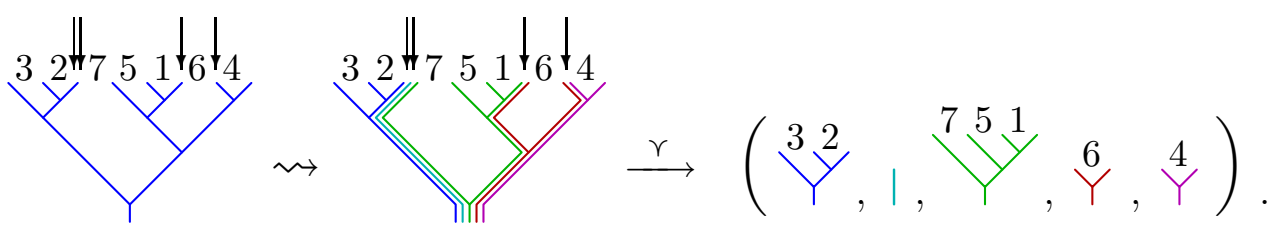

Given $v \in \mathfrak{S}_{m}$ and an ordered forest $\left(w_{0}, \ldots, w_{m}\right)$, let $\left(w_{0}, \ldots, w_{m}\right) / v$ be the ordered tree obtained by grafting the root of $w_{i}$ to the $i$ th leaf of $v$, where the nodes of $v$ are greater than all nodes of $w$, but the relative orders within the $w_{i}$ and $v$ are maintained. When $v$ is the ordered tree corresponding to 1432 and $w \stackrel{\curlyvee}{\rightarrow}\left(w_{0}, \ldots, w_{m}\right)$ is the splitting (1.1), this grafting is

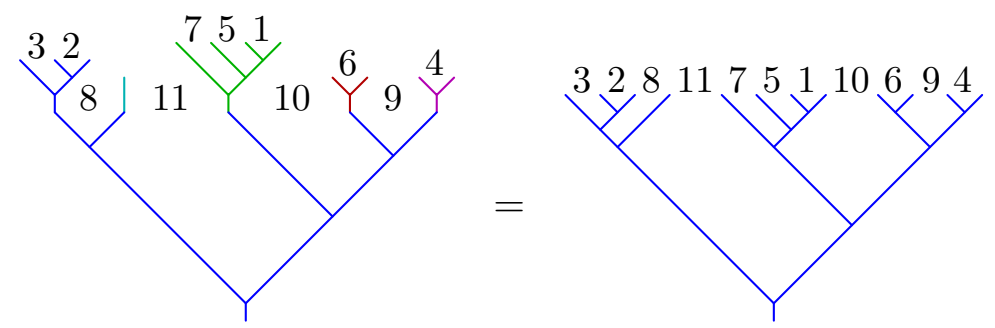

The notions of splitting and grafting also make sense for the unordered trees $\mathcal{Y}_{n}$ and we use the same notation, $\stackrel{\bullet}{\rightarrow}$ • and $\bullet / \bullet$. (Simply delete the labels in the constructions above.) These operations of splitting and grafting are compatible with the map $\tau: \mathfrak{S}_{.} \rightarrow \mathcal{Y}_{\bullet}:$ if $w \stackrel{r}{\rightarrow}\left(w_{0}, \ldots, w_{m}\right)$ then $\tau(w) \stackrel{r}{\rightarrow}\left(\tau\left(w_{0}\right), \ldots, \tau\left(w_{m}\right)\right)$ and all splittings in $\mathcal{Y}$. are induced in this way from splittings in $\mathfrak{S}$.. The same is true for grafting, $\tau\left(\left(w_{0}, \ldots, w_{m}\right) / v\right)=\left(\tau\left(w_{0}\right), \ldots, \tau\left(w_{m}\right)\right) / \tau(v)$.

1.2. SSym and YSym. For basics on Hopf algebras, see [19]. Let $\mathfrak{S} S y m:=$ $\bigoplus_{n \geq 0} \mathfrak{S} S y m_{n}$ be the graded $\mathbb{Q}$-vector space whose $n^{\text {th }}$ graded piece has basis $\left\{F_{w} \mid w \in \mathfrak{S}_{n}\right\}$. Malvenuto and Reutenauer [16] defined a Hopf algebra structure on $\mathfrak{S}$ Sym. For $w \in \mathfrak{S}$., define the coproduct

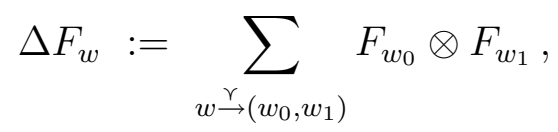

where $\left(w_{0}, w_{1}\right)$ is a pair of ordered trees. If $v \in \mathfrak{S}_{m}$, define the product

$$
F_{w} \cdot F_{v}:=\sum_{w \stackrel{\curlyvee}{\rightarrow}\left(w_{0}, \ldots, w_{m}\right)} F_{\left(w_{0}, \ldots, w_{m}\right) / v} .
$$

The counit is the projection $\varepsilon: \mathfrak{S} S y m \rightarrow \mathfrak{S} S y m_{0}$ onto the 0th graded piece, which is spanned by the unit, $1=F_{\emptyset}$, for this multiplication.

Proposition 1.1 ([16]). With these definitions of coproduct, product, counit, and unit, $\mathfrak{S} S y m$ is a graded, connected cofree Hopf algebra that is neither commutative nor cocommutative. 
Let $\mathcal{Y}$ Sym $:=\bigoplus_{n>0} \mathcal{Y} S y m_{n}$ be the graded $\mathbb{Q}$-vector space whose $n^{\text {th }}$ graded piece has basis $\left\{F_{t} \mid t \in \mathcal{Y}_{n}\right\}$. Loday and Ronco [14] defined a Hopf algebra structure on $\mathcal{Y}$ Sym. For $t \in \mathcal{Y}_{\bullet}$, define the coproduct

$$
\Delta F_{t}:=\sum_{\substack{\curlyvee \\ t \rightarrow\left(t_{0}, t_{1}\right)}} F_{t_{0}} \otimes F_{t_{1}},
$$

and if $s \in \mathcal{Y}_{m}$, define the product

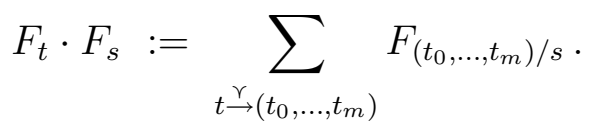

The counit is the projection $\varepsilon: \mathcal{Y} S y m \rightarrow \mathcal{Y} S y m_{0}$ onto the 0th graded piece, which is spanned by the unit, $1=F_{1}$, for this multiplication. The map $\tau$ extends to a linear map $\boldsymbol{\tau}: \mathfrak{S} S y m \rightarrow \mathcal{Y}$ Sym, defined by $\boldsymbol{\tau}\left(F_{w}\right)=F_{\tau(w)}$.

Proposition 1.2 ([14]). With these definitions of coproduct, product, counit, and unit, $\mathcal{Y}$ Sym is a graded, connected cofree Hopf algebra that is neither commutative nor cocommutative and the map $\boldsymbol{\tau}$ a morphism of Hopf algebras.

Some structures of the Hopf algebras SSym and Y Sym, particularly their primitive elements and coradical filtrations are better understood with respect to a second basis. The Möbius function $\mu$ (or $\mu_{P}$ ) of a poset $P$ is defined for pairs $(x, y)$ of elements of $P$ with $\mu(x, y)=0$ if $x \nless y, \mu(x, x)=1$, and, if $x<y$, then

$$
\mu(x, y)=-\sum_{x \leq z<y} \mu(x, z) \quad \text { so that } \quad 0=\sum_{x \leq z \leq y} \mu(x, z) .
$$

For $w \in \mathfrak{S}_{\text {. }}$ and $t \in \mathcal{Y}_{\text {. }}$, set

$$
M_{w}:=\sum_{w \leq v} \mu(w, v) F_{v} \quad \text { and } \quad M_{t}:=\sum_{t \leq s} \mu(t, s) F_{s},
$$

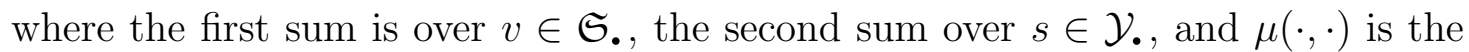
Möbius function in the weak and Tamari orders.

Proposition $1.3([1,2])$. If $w \in \mathfrak{S}$., then

$$
\boldsymbol{\tau}\left(M_{w}\right)= \begin{cases}M_{\tau(w)}, & \text { if } w=\max (\tau(w)), \\ 0, & \text { otherwise }\end{cases}
$$

and

$$
\Delta M_{w}=\sum_{w=u \backslash v} M_{u} \otimes M_{v}
$$

If $t \in \mathcal{Y}_{\bullet}$, then

$$
\Delta M_{t}=\sum_{t=r \backslash s} M_{r} \otimes M_{s} .
$$

This implies that the set $\left\{M_{w} \mid w \in \mathfrak{S}\right.$. is indecomposable $\}$ is a basis for the primitive elements of $\mathfrak{S} S y m$ (and the same for $\mathcal{Y} S y m$ ), thereby explicitly realizing the cofree-ness of $\mathfrak{S}$ Sym and $\mathcal{Y}$ Sym. 
1.3. Möbius functions and interval retracts. A pair $f: P \rightarrow Q$ and $g: Q \rightarrow P$ of poset maps is a Galois connection if $f$ is left adjoint to $g$ in that

$$
\forall p \in P \text { and } q \in Q, \quad f(p) \leq_{Q} q \Longleftrightarrow p \leq_{P} g(q) .
$$

When this occurs, Rota [21, Theorem 1] related the Möbius functions of $P$ and $Q$ :

$$
\forall p \in P \text { and } q \in Q, \quad \sum_{f(y)=q} \mu_{P}(p, y)=\sum_{g(x)=q} \mu_{Q}(x, q) .
$$

Rota's formula was used in [2] to establish the coproduct formulas (1.4) and (1.6), as the maps $\tau: \mathfrak{S}$. $\rightarrow \mathcal{Y}$. and max: $\mathcal{Y}$. $\rightarrow \mathfrak{S}$. form a Galois connection $[4$, Section 9].

We do not have a Galois connection between $\mathfrak{S}_{\text {. and }} \mathcal{M}_{\text {. }}$, and so cannot use Rota's formula. Nevertheless, there is a useful relation between the Möbius functions of $\mathfrak{S}$. and $\mathcal{M}$. that we establish here in a general form. A surjective poset map $f: P \rightarrow Q$ from a finite lattice $P$ is an interval retract if the fibers of $f$ are intervals and if $f$ admits an order-preserving section $g: Q \rightarrow P$ with $f \circ g=$ id.

Theorem 1.4. Let the poset map $f: P \rightarrow Q$ is an interval retract, then the Möbius functions $\mu_{P}$ and $\mu_{Q}$ of $P$ and $Q$ are related by the formula

$$
\mu_{Q}(x, y)=\sum_{\substack{f(a)=x \\ f(b)=y}} \mu_{P}(a, b) \quad(\forall x, y \in Q) .
$$

In Section 2, we define an interval retract $\beta: \mathfrak{S}_{n} \rightarrow \mathcal{M}_{n}$.

We evaluate each side of (1.7) using Hall's formula, which expresses the Möbius function in terms of chains. A linearly ordered subset $C: x_{0}<\cdots<x_{r}$ of a poset is a chain of length $\ell(C)=r$ from $x_{0}$ to $x_{r}$. Given a poset $P$, let $\mathrm{\Psi}(P)$ be the set of all chains in $P$. A poset $P$ is an interval if it has a unique maximum element and a unique minimum element. If $P=[x, y]$ is an interval, let $\mathrm{Y}^{\prime}(P)$ denote the chains in $P$ beginning in $x$ and ending in $y$. Hall's formula states that

$$
\mu(x, y)=\sum_{C \in \mathrm{U}^{\prime}[x, y]}(-1)^{\ell(C)} .
$$

Our proof rests on the following two lemmas.

Lemma 1.5. If $P$ is an interval, then $\sum_{C \in \mathrm{\Psi}(P)}(-1)^{\ell(C)}=1$.

Proof. Suppose that $P=[x, y]$ and append new minimum and maximum elements to $\mathrm{P}$ to get $\hat{P}:=P \cup\{\hat{0}, \hat{1}\}$. Then the definition of Möbius function (1.2) gives

$$
\mu(\hat{0}, \hat{1})=-\sum_{\hat{0} \leq z \leq y} \mu(\hat{0}, z),
$$

which is zero by (1.2). By Hall's formula,

$$
0=\mu(\hat{0}, \hat{1})=\sum_{C \in \mathrm{\Psi}^{\prime}[\hat{0}, \hat{1}]}(-1)^{\ell(C)}=-1+\sum_{C \in \mathrm{\Psi}(P)}(-1)^{\ell(C)+2},
$$

where the term -1 comes from the chain $\hat{0}<\hat{1}$. This proves the lemma. 
Call a partition $P=K_{0} \sqcup \cdots \sqcup K_{r}$ of $P$ into subposets $K_{i}$ monotone if $x<y$ with $x \in K_{i}$ and $y \in K_{j}$ implies that $i \leq j$. Given $\emptyset \subsetneq I \subseteq[0, r]$, write $\Psi_{I}(P)$ for the subset of chains $C$ in $\mathrm{\Psi}(P)$ such that $C \cap K_{i} \neq \emptyset$ if and only if $i \in I$.

Lemma 1.6. Let $P=K_{0} \sqcup \cdots \sqcup K_{r}$ be a monotonic partition of a poset $P$. If $\bigcup_{i \in I} K_{i}$ is an interval for all $I \subseteq[0, r]$, then

$$
\sum_{C \in \mathrm{U}_{[0, r]}(P)}(-1)^{\ell(C)}=(-1)^{r} .
$$

Proof. We argue by induction on $r$. Lemma 1.5 is the case $r=0$ (wherein $K_{0}=P$ ), so we consider the case $r \geq 1$.

Form the poset $\hat{P}=P \cup\{\hat{0}, \hat{1}\}$ as in the proof of Lemma 1.5. Since $P$ is an interval, we have $\sum_{C \in \mathrm{U}^{\prime}[\hat{0}, \hat{1}]}(-1)^{\ell(C)}=0$. As $\mathbf{\Psi}^{\prime}[\hat{0}, \hat{1}]=\bigsqcup_{I} \mathrm{\Psi}_{I}(P)$ we have,

$$
0=-1+\sum_{\emptyset \subsetneq I \subsetneq[0, r]}\left(\sum_{C \in \mathrm{U}_{I}(P)}(-1)^{\ell(C)}\right)+\sum_{C \in \mathrm{U}_{[0, r]}(P)}(-1)^{\ell(C)}
$$

where the term -1 counts the chain $\hat{0}<\hat{1}$. Applying induction, we have

$$
0=\sum_{k=0}^{r}\left(\begin{array}{c}
r+1 \\
k
\end{array}\right)(-1)^{k-1}+\sum_{C \in \mathrm{U}_{[0, r]}(P)}(-1)^{\ell(C)} .
$$

Comparing this to the binomial expansion of $(1-1)^{r+1}$ completes the proof.

Proof of Theorem 1.4. Fix $x<y$ in $Q$. We use Hall's formula to rewrite the righthand side of (1.7) as

$$
\sum_{\substack{f(x)=a \\ f(y)=b}} \sum_{C \in \mathrm{Y}^{\prime}[a, b]}(-1)^{\ell(C)}
$$

Fix a chain $D: q_{0}<\cdots<q_{r}$ in $\mathrm{Y}^{\prime}[x, y]$ and let $\left.P\right|_{D}$ be the subposet of $P$ consisting of elements that occur in some chain of $P$ that maps to $D$ under $f$. This is nonempty as $f$ has section. Furthermore, the sets $K_{i}:=\left.f^{-1}\left(q_{i}\right) \cap P\right|_{D}$, for $i=$ $0, \ldots, r$, form a monotonic partition of $\left.P\right|_{D}$. We claim that $\bigcup_{i \in I} K_{i}$ is an interval for all $I \subseteq[0, r]$. If so, let us first rewrite (1.9) as a sum over chains $D$ in $Q$,

$$
\sum_{D \in \mathrm{U}^{\prime}[x, y]} \sum_{C \in \mathrm{U}_{[0, \ell(D)]}\left(\left.P\right|_{D}\right)}(-1)^{\ell(C)} .
$$

By Lemma 1.6, the inner sum becomes $\sum_{D}(-1)^{\ell(D)}$, which completes the proof.

To prove the claim, suppose that $I=\left\{i_{0}<\cdots<i_{s}\right\}$. Each set $K_{i}(i \in I)$ is an interval, as it is the intersection of two intervals in the lattice $P$. Thus $K_{i_{0}}$ and $K_{i_{s}}$ are intervals with minimum and maximum elements $m$ and $M$, respectively. Any chain in $\bigcup_{i \in I} K_{i}$ can be extended to a chain beginning with $m$ and ending at $M$, so $\bigcup_{i \in I} K_{i}$ is an interval. 


\section{The Multiplihedra $\mathcal{M}$.}

The map $\tau: \mathfrak{S}$. $\rightarrow \mathcal{Y}$. forgets the linear ordering of the node poset of an ordered tree, and it induces a morphism of Hopf algebras $\boldsymbol{\tau}: \mathfrak{S S y m} \rightarrow \mathcal{Y}$ Sym. In fact, one may take the (ahistorical) view that the Hopf structure on $\mathcal{Y} S y m$ is induced from that on $\mathfrak{S} S y m$ via the map $\tau$. Forgetting some, but not all, of the structure on a tree in $\mathfrak{S}$. factorizes the map $\tau$. Here, we study combinatorial consequences of one such factorization, and later treat its algebraic consequences.

2.1. Bi-leveled trees. A bi-leveled tree $(t ; \mathrm{T})$ is a planar binary tree $t \in \mathcal{Y}_{n}$ together with an (upper) order ideal $T$ of its node poset, where $T$ contains the leftmost node of $t$ as a minimal element. Thus $\mathrm{T}$ contains all nodes along the path from the leftmost leaf to the root, and none above the leftmost node. Numbering the gaps between the leaves of $t$ by $1, \ldots, n$ from left to right, $\mathrm{T}$ becomes a subset of $\{1, \ldots, n\}$.

Saneblidze and Umble [22] introduced bi-leveled trees to describe a cellular projection from the permutahedra to Stasheff's multiplihedra $\mathcal{M}_{\text {. }}$, with the bi-leveled trees on $n$ nodes indexing the vertices $\mathcal{M}_{n}$. Stasheff used a different type of tree for the vertices of $\mathcal{M}$. . These alternative trees lead to a different Hopf structure which we explore in a forthcoming paper [9]. We remark that $\mathcal{M}_{0}=\{\mid\}$.

The partial order on $\mathcal{M}_{n}$ is defined by $(s ; \mathrm{S}) \leq(t ; \mathrm{T})$ if $s \leq t$ in $\mathcal{Y}_{n}$ and $\mathrm{S} \supseteq \mathrm{T}$. The Hasse diagrams of the posets $\mathcal{M}_{n}$ are 1-skeleta for the multiplihedra. We represent a bi-leveled tree by drawing the underlying tree $t$ and circling the nodes in $\mathrm{T}$. The Hasse diagram of $\mathcal{M}_{4}$ appears in Figure 2.

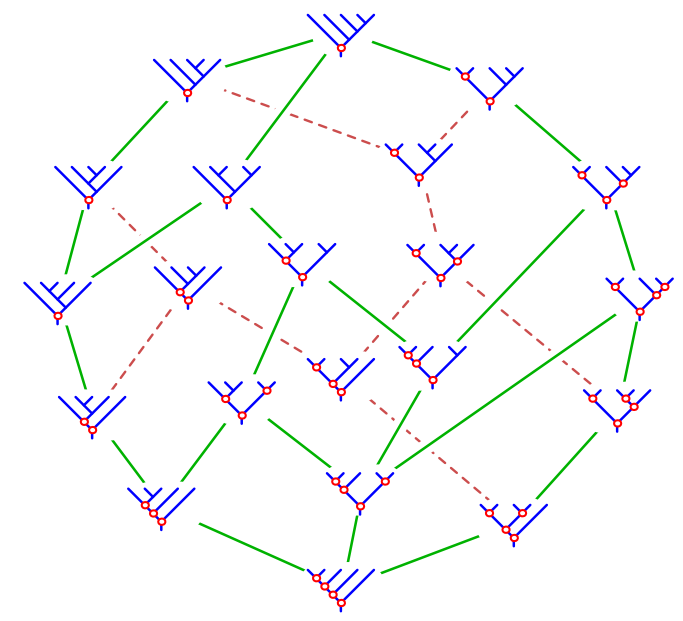

Figure 2. The 1-skeleton of the multiplihedron $\mathcal{M}_{4}$.

2.2. Poset maps. Forgetting the order ideal in a bi-leveled tree, $(t ; \mathrm{T}) \mapsto t$, is a poset $\operatorname{map} \phi: \mathcal{M}$. $\rightarrow \mathcal{Y}_{\text {. }}$. We define a map $\beta: \mathfrak{S}$. $\rightarrow \mathcal{M}$. so that

$$
\text { S. } \stackrel{\beta}{\longrightarrow} \text { M. } \stackrel{\phi}{\longrightarrow} \mathcal{Y} \text {. }
$$

factors the map $\tau: \mathfrak{S}$. $\rightarrow \mathcal{Y}_{\bullet}$, and we define a right inverse (section) $\iota$ of $\beta$. 
Let $w \in \mathfrak{S}$. be an ordered tree. Define the set

$$
\mathrm{T}(w):=\{i \mid w(i) \geq w(1)\} .
$$

Observe that $(\tau(w) ; \mathrm{T}(w))$ is a bi-leveled tree. Indeed, as $w$ is a linear extension of $\tau(w), \mathbf{T}(w)$ is an upper order ideal which by definition (2.1) contains the leftmost node as a minimal element. Since covers in the weak order can only decrease the subset $\mathrm{T}(w)$ and $\tau$ is also a poset map, we see that $\beta$ is a poset map.

Theorem 2.1. The maps $\beta:$ S. $\rightarrow \mathcal{M}$. and $\phi: \mathcal{M}$. $\rightarrow \mathcal{Y}$. are surjective poset maps with $\tau=\phi \circ \beta$.

The fibers of the map $\beta$ are intervals (indeed, products of intervals); see Figure 3. We prove this using an equivalent representation of a bi-leveled tree and

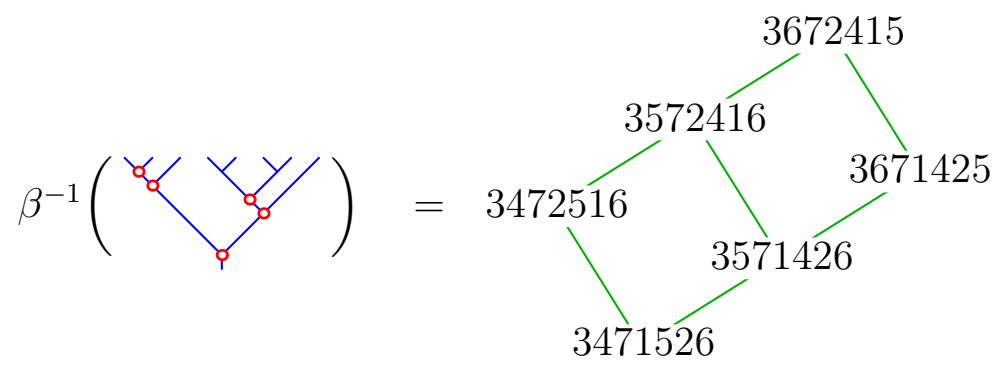

Figure 3. The preimages of $\beta$ are intervals.

a description of the map $\beta$ in that representation. If we prune a bi-leveled tree $b=(t ; \mathrm{T})$ above the nodes in $\mathrm{T}$ (but not on the leftmost branch) we obtain a tree $t_{0}^{\prime}$ (the order ideal) on $r$ nodes and a planar forest $\boldsymbol{t}=\left(t_{1}, \ldots, t_{r}\right)$ of $r$ trees. If we prune $t_{0}^{\prime}$ just below its leftmost node, we obtain the tree $Y$ (from the pruning) and a tree $t_{0}$, and $t_{0}^{\prime}$ is obtained by grafting $Y$ onto the leftmost leaf of $t_{0}$. We may recover $b$ from this tree $t_{0}$ on $r-1$ nodes and the planar forest $\boldsymbol{t}=\left(t_{1}, \ldots, t_{r}\right)$, and so we also write $b=\left(t_{0}, \boldsymbol{t}\right)$. We illustrate this correspondence in Figure 4.

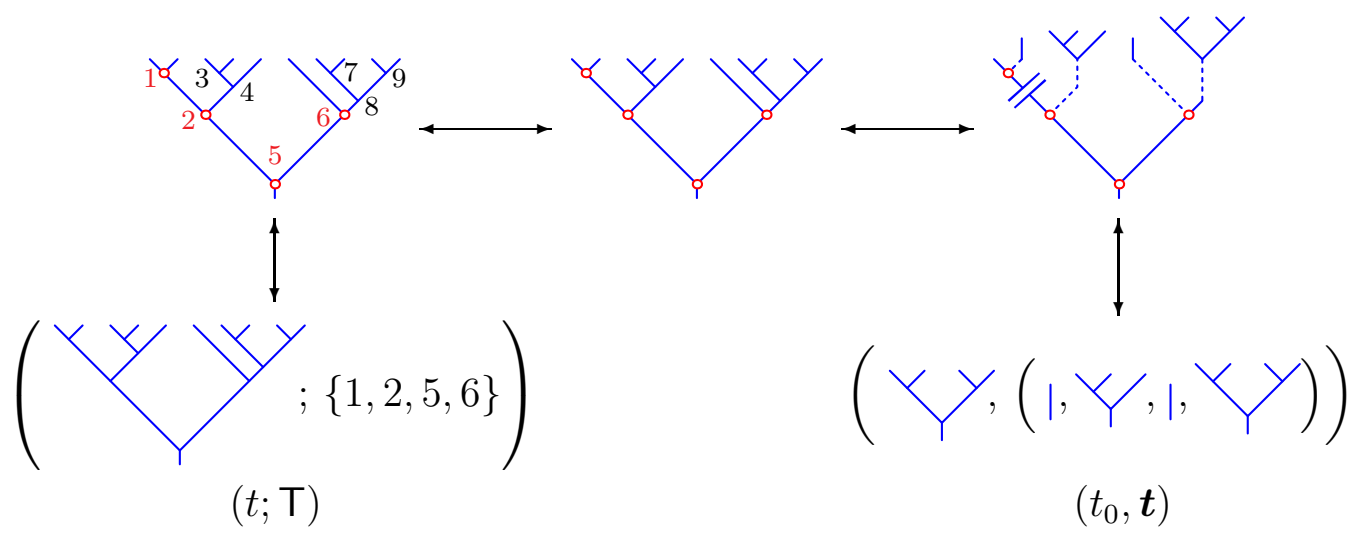

Figure 4. Two representations of bi-leveled trees.

We describe the map $\beta$ in terms of this second representation of bi-leveled trees. Given a permutation $w$ with $\beta(w)=\left(t\right.$; T) and $|\mathrm{T}|=r$, let $u_{1} u_{2} \ldots u_{r}$ be the 
restriction of $w$ to the set T. We may write the values of $w$ as $w=u_{1} v^{1} u_{2} \cdots u_{r} v^{r}$, where $v^{i}$ is the (possibly empty) subword of $w$ between the numbers $u_{i}$ and $u_{i+1}$ and $v^{r}$ is the word after $u_{r}$. Call this the bi-leveled factorization of $w$. For example,

$$
654789132 \longmapsto \begin{gathered}
(6,54,7, \emptyset, 8, \emptyset, 9,132) . \\
u_{1} \quad v^{1} \quad u_{2} \quad v^{2} \quad u_{3} v^{3} \quad u_{4} v^{4}
\end{gathered}
$$

Note that $\beta(w)=\left(\tau\left(\overline{u_{2} \ldots u_{r}}\right),\left(\tau\left(\overline{v^{1}}\right), \ldots, \tau\left(\overline{v^{r}}\right)\right)\right)$.

Theorem 2.2. For any $b \in \mathcal{M}_{n}$ the fiber $\beta^{-1}(b) \subseteq \mathfrak{S}_{n}$ is a product of intervals.

Proof. Let $b=\left(t_{0},\left(t_{1}, \ldots, t_{r}\right)\right)=(t ; \mathrm{T}) \in \mathcal{M}_{n}$ be a bi-leveled tree. A permutation $w \in \beta^{-1}(b) \in \mathfrak{S}_{n}$ has a bi-leveled factorization $w=u_{1} v^{1} u_{2} \ldots u_{r} v^{r}$ with

(i) $\left.\quad w\right|_{\mathrm{T}}=u_{1} u_{2} \ldots u_{r}, u_{1}=n+1-r, \tau\left(\overline{u_{2} \ldots u_{r}}\right)=t_{0}$, and

(ii) $\tau\left(\overline{v^{i}}\right)=t_{i}$, for $i=1, \ldots, r$.

Since $u_{1}<u_{2}, \ldots, u_{r}$ are the values of $w$ in the positions of $\mathrm{T}$, and $u_{1}=n+1-r$ exceeds all the letters in $v^{1}, \ldots, v^{r}$, which are the values of $w$ in the positions in the complement of $\mathrm{T}$, these two parts of the bi-leveled factorization may be chosen independently to satisfy $(2.2)$, which shows that $\beta^{-1}(b)$ is a product.

To see that the factors are intervals, and thus $\beta^{-1}(b)$ is an interval, we examine the conditions $(i)$ and $(i i)$ separately. Those $u_{1} \ldots u_{r}=\left.w\right|_{\mathrm{T}}$ for $w$ in the fiber $\beta^{-1}(b)$ are exactly the set of $n+1-r, u_{2}, \ldots, u_{r}$ with $\left\{u_{2}, \ldots, u_{r}\right\}=\{n+2-r, \ldots, n\}$ and $\tau\left(\overline{u_{2} \ldots u_{r}}\right)=t_{0}$. This is a poset under the restriction of the weak order, and it is in natural bijection with the interval $\tau^{-1}\left(t_{0}\right) \subset \mathfrak{S}_{r-1}$. Its minimal element is $\min _{0}(b)=$ $u_{1} u_{2} \ldots u_{r}$, where $u_{2} \ldots u_{r}$ is the unique 231-avoiding word on $\{n+1-r, \ldots, n\}$ satisfying $(i)$, and its maximal element is $\max _{0}(b)=u_{1} u_{2} \ldots u_{r}$, where now $u_{2} \ldots u_{r}$ is the unique 132 -avoiding word on $\{n+1-r, \ldots, n\}$ satisfying $(i)$.

Now consider sequences of words $v^{1}, \ldots, v^{r}$ on distinct letters $\{1, \ldots, n-r\}$ satisfying ( $i i)$. This is also a poset under the restriction of the weak order. It has a minimal element, which is the unique such sequence $\min (b)$ satisfying $(i i)$ where the letters of $v^{i}$ preceed those of $v^{j}$ whenever $i<j$, and where each $v^{i}$ is 231-avoiding. Its maximal element is the unique sequence $\max (b)$ satisfying $(i i)$ where the letters of $v^{i}$ are greater than those of $v^{j}$ when $i<\bar{j}$ and $v^{i}$ is 132-avoiding.

The fibers of $\beta$ are intervals so that consistently choosing the minimum or maximum in a fiber gives two set-theoretic sections. These are not order-preserving as may be seen from Figure 5. We have $\psi<<\psi$ but the maxima in their fibers

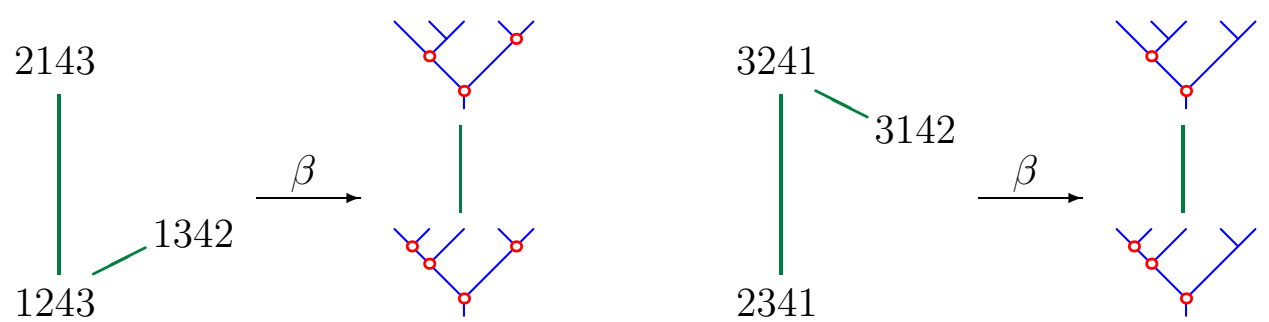

FiguRE 5. Fibers of $\beta$. 
under $\beta, 1342$ and 2143, are incomparable. Similarly, $y<<y$ but the minima in their fibers under $\beta, 2341$ and 3142, are incomparable. This shows that the map $\beta:$ S. $\rightarrow \mathcal{M}$. is not a lattice congruence (unlike the map $\tau: \mathfrak{S}$. $\rightarrow \mathcal{Y}$. [20]).

In the notation of the proof, given a bi-leveled tree $b=\left(t_{0},\left(t_{1}, \ldots, t_{r}\right)\right)$, let $\iota(b)$ be the permutation $w \in \beta^{-1}(b)$ with bi-leveled factorization $w=u_{1} v^{1} u_{2} \ldots u_{r} v^{r}$ where $u_{1} u_{2} \ldots u_{r}=\min _{0}(b)$ and $\left(v^{1}, \ldots, v^{r}\right)=\underline{\max }(b)$. This defines a map $\iota: \mathcal{M}_{n} \rightarrow \mathfrak{S}_{n}$ that is a section of the map $\beta$. For example,

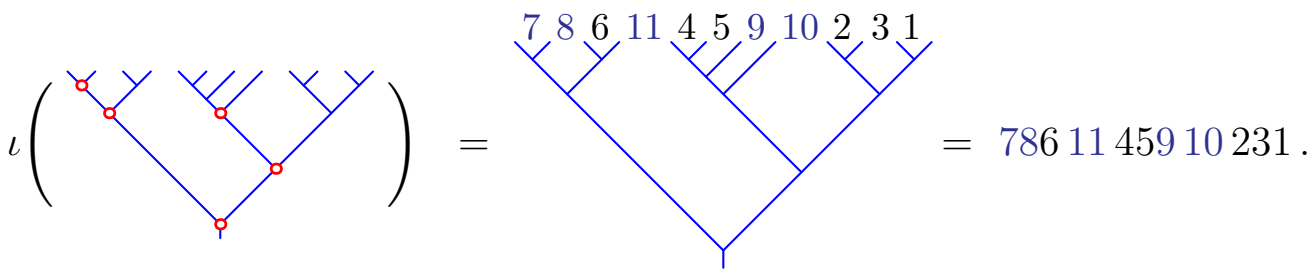

Remark 2.3. This map $\iota$ may be characterized in terms of pattern avoidance: the permutation $\iota(b)$ is the unique $w \in \beta^{-1}(b)$ avoiding the pinned patterns

$$
\{\underline{2} 031, \underline{0} 231, \underline{3} 021\} \text {, }
$$

where the underlined letter must be the first letter of a permutation. To see this, note that the first pattern forces the letters in $v^{i}$ to be larger than those in $v^{i+1}$ for $1 \leq i<r$, the second pattern forces $u_{2} \ldots u_{r}$ to be 231-avoiding, and the last pattern forces each $v^{i}$ to be 132-avoiding.

Theorem 2.4. The map $\iota$ is injective, right-inverse to $\beta$, and order-preserving. That is, $\beta: \mathfrak{S}_{n} \rightarrow \mathcal{M}_{n}$ is an interval retract.

Since $\mathfrak{S}_{n}$ is a lattice [10], the fibers of $\beta$ are intervals, and $\iota$ is a section of $\beta$. That is, we need only verify that $\iota$ is order-preserving. We begin by describing the covers in $\mathcal{M}_{\text {. }}$. Since $\beta$ is a surjective poset map, every cover in $\mathcal{M}_{n}$ is the image of some cover $w \lessdot w^{\prime}$ in $\mathfrak{S}_{n}$.

Lemma 2.5. If a cover $w \lessdot w^{\prime} \in \mathfrak{S}_{n}$ does not collapse under $\beta$, i.e., $\beta(w) \neq \beta\left(w^{\prime}\right)$, then it yields one of three types of covers $\beta(w) \lessdot \beta\left(w^{\prime}\right)$ in $\mathcal{M}_{n}$.

(i) In exactly one tree $t_{i}$ in $\beta(w)=\left(t_{0},\left(t_{1}, \ldots, t_{r}\right)\right)$, a node is moved from left to right across its parent to obtain $\beta\left(w^{\prime}\right)$. That is, $t_{i} \lessdot t_{i}^{\prime}$.

(ii) If $\beta(w)=(t ; \mathrm{T})$, the leftmost node of $t$ is moved across its parent, which has no other child in the order ideal $\mathrm{T}$, and is deleted from $\mathrm{T}$ to obtain $\beta\left(w^{\prime}\right)$.

(iii) If $\mathrm{T}(w)=\left\{1=T_{1}<\cdots<T_{r}\right\}$, then $\tau\left(w^{\prime}\right)=\tau(w)$ and $\mathrm{T}\left(w^{\prime}\right)=\mathrm{T}(w) \backslash\left\{T_{j}\right\}$ for some $j>2$.

Proof. Put $w^{\prime}=(k, k+1) w$, with $k, k+1$ appearing in order in $w$. Let $(t ; \mathrm{T})$ and $\left(t_{0},\left(t_{1}, \ldots, t_{r}\right)\right)$ be the two representations of $\beta(w)$. Write $\mathrm{T}=\left\{T_{1}<\cdots<T_{r}\right\}$ (with $T_{1}=1$ ) and $\left.w\right|_{\mathrm{T}}=u_{1} u_{2} \ldots u_{r}$. If $w \lessdot w^{\prime}$ and $\beta(w) \lessdot \beta\left(w^{\prime}\right)$, then $k$ appears within $w$ in one of three ways: $(i) u_{1} \neq k$, (ii) $u_{1}=k$ and $u_{2}=k+1$, or $($ iii $)$ $u_{1}=k$ and $u_{j}=k+1$ for some $j>2$. These yield the corresponding descriptions in the statement of the lemma. (Note that in type $(i), \mathrm{T}\left(w^{\prime}\right)=\mathrm{T}$, so if we set $\beta\left(w^{\prime}\right)=\left(t_{0}^{\prime},\left(t_{1}^{\prime}, \ldots, t_{r}^{\prime}\right)\right)$, then $t_{i}=t_{i}^{\prime}$, except for one index $i$, where $t_{i} \lessdot t_{i}^{\prime}$.) 


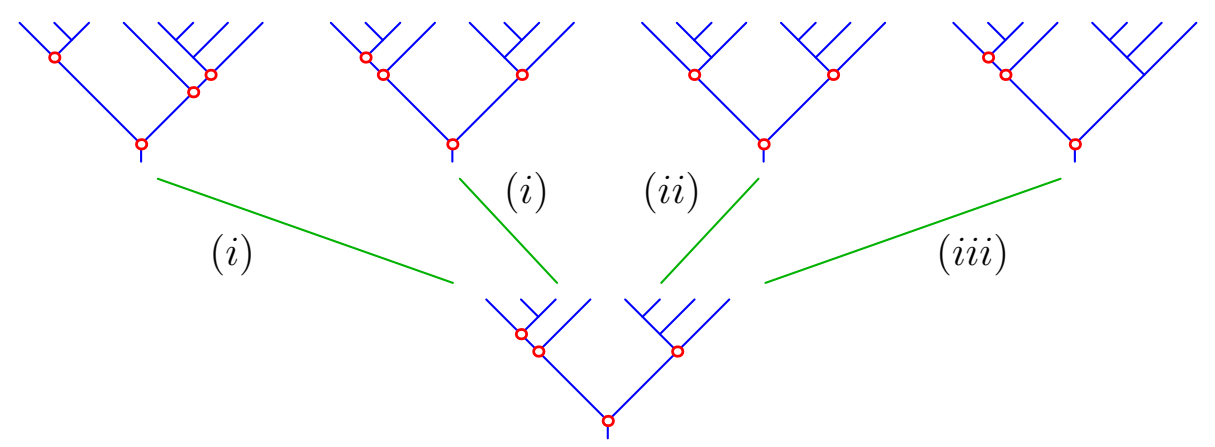

Figure 6. Some covers in $\mathcal{M}_{7}$.

Figure 6 illustrates these three types of covers, labeled by their type.

For $\mathrm{T} \subset\{1, \ldots, n\}$ with $1 \in \mathrm{T}$, let $\mathfrak{S}_{n}(\mathrm{~T}):=\left\{w \in \mathfrak{S}_{n} \mid \mathrm{T}(w)=\mathrm{T}\right\}$. Let $\mathcal{M}_{n}(\mathrm{~T})$ be those bi-leveled trees whose order ideal consists of the nodes in T. Note that $\beta\left(\mathfrak{S}_{n}(\mathrm{~T})\right)=\mathcal{M}_{n}(\mathrm{~T})$ and $\beta^{-1}\left(\mathcal{M}_{n}(\mathrm{~T})\right)=\mathfrak{S}_{n}(\mathrm{~T})$.

Lemma 2.6. The map $\iota: \mathcal{M}_{n}(\mathrm{~T}) \rightarrow \mathfrak{S}_{n}(\mathrm{~T})$ is a map of posets.

Proof. Let $\mathrm{T}=\left\{1=T_{1}<\cdots<T_{r}\right\}$. Setting $T_{r+1}=n+1$, define $a_{i}:=T_{i+1}-T_{i}-1$ for $i=1, \ldots, r$. Then $b \mapsto\left(t_{0},\left(t_{1}, \ldots, t_{r}\right)\right)$ gives an isomorphism of posets,

$$
\mathcal{M}_{n}(\mathrm{~T}) \stackrel{\sim}{\longrightarrow} \mathcal{Y}_{r-1} \times \mathcal{Y}_{a_{1}} \times \cdots \times \mathcal{Y}_{a_{r}} .
$$

As the maps min, max: $\mathcal{Y}_{a} \rightarrow \mathfrak{S}_{a}$ are order-preserving, the proof of Theorem 2.2 gives the desired result.

Proof of Theorem 2.4. Let $b \lessdot c$ be a cover in $\mathcal{M}_{n}$. We will show that $\iota(b) \leq \iota(c)$ in $\mathfrak{S}_{n}$. Suppose that $b=(t ; \boldsymbol{\top})$, with $\mathbf{T}=\left\{1=T_{1}<\cdots<T_{r}\right\}$. Let $\iota(b)$ have bi-leveled factorization $\iota(b)=u_{1} v^{1} u_{2} v^{2} \ldots u_{r} v^{r}$, and set $k:=n+1-|\mathrm{T}|$.

The result is immediate if the cover $b \lessdot c$ is of type $(i)$, for then $b, c \in \mathcal{M}_{n}(\mathrm{~T})$ and $\iota: \mathcal{M}_{n}(\mathbf{T}) \rightarrow \mathfrak{S}_{n}$ is order-preserving, as observed in Lemma 2.6.

Now suppose that $b \lessdot c$ is a cover of type $(i i)$. Set $w:=\iota(b)$. We claim that $w \lessdot(k, k+1) w$ and $\iota(c)=(k, k+1) w$. Now, $u_{1}=k$ labels the leftmost node of $b$, so the first claim is immediate. Note that $u_{2}$ labels the parent of the node labeled $b$. This parent has no other child in $\mathrm{T}$, so we must have $u_{2}<u_{3}$. As $u_{2} u_{3} \ldots u_{r}$ is 231-avoiding and contains $k+1$, we must have $u_{2}=k+1$. This shows that

$$
\iota(c)=(k, k+1) w=u_{2}\left(v^{1} u_{1} v^{2}\right) u_{3} \ldots u_{r} v^{r} .
$$

Indeed, $u_{2}$ is minimal among $u_{2}, \ldots, u_{r}$ and $u_{3} \ldots u_{r}$ is 231-avoiding, thus $\min _{0}(c)=$ $u_{2} \ldots u_{r}$. The bi-leveled factorization of $(k, k+1) w$ gives $\left(v^{1} u_{1} v^{2}, v^{3}, \ldots, v^{r}\right)$, which we claim is $\max (c)$. As $u_{1}$ is the largest letter in the sequence, we need only check that $v^{1} u_{1} v^{2}$ is 132-avoiding. But this is true for $v^{1}$ and $v^{2}$ and there can be no 132-pattern involving $u_{1}$ as the letters in $v^{1}$ are all greater than those in $v^{2}$.

Finally, suppose that $b \lessdot c$ is of type $\left(\right.$ iii). Then $c=\left(t\right.$; $\left.\top \backslash\left\{T_{j}\right\}\right)$ for some $j>2$. We will find a permutation $w^{\prime} \in \beta^{-1}(b)$ satisfying $(k, k+1) w^{\prime} \in \beta^{-1}(c)$ and

$$
\iota(b) \leq w^{\prime} \lessdot(k, k+1) w^{\prime} \leq \iota(c) .
$$


Let $w^{\prime} \in \beta^{-1}(b)$ be the minimal permutation having bi-leveled factorization

$$
w^{\prime}=u_{1}^{\prime} v^{1} u_{2}^{\prime} \ldots u_{r}^{\prime} v^{r}, \quad \text { with } \quad u_{j}^{\prime}=k+1 .
$$

Here $\left(v^{1}, \ldots, v^{r}\right)=\underline{\max }(b)$ is the same sequence as in $\iota(b)$. The structure of $\beta^{-1}(b)$ implies that $\iota(b) \leq w^{\prime}$. We also have

$$
w^{\prime} \lessdot(k, k+1) w^{\prime} \quad \text { and } \quad \beta\left((k, k+1) w^{\prime}\right)=c .
$$

While $\iota(c)$ and $(k, k+1) w^{\prime}$ are not necessarily equal, we do have that

$$
\left.(k, k+1) w^{\prime}\right|_{T \backslash\left\{T_{j}\right\}}=u_{j}^{\prime} u_{2}^{\prime} \ldots u_{j-1}^{\prime} u_{j+1}^{\prime} \ldots u_{r}^{\prime}
$$

and $u_{2}^{\prime} \ldots u_{j-1}^{\prime} u_{j+1}^{\prime} \ldots u_{r}^{\prime}$ is 231-avoiding. That is, $\left.(k, k+1) w^{\prime}\right|_{\mathbb{T} \backslash\left\{T_{j}\right\}}=\left.\iota(c)\right|_{\mathbb{T} \backslash\left\{T_{j}\right\}}$. Otherwise, $w^{\prime}$ would not be minimal. The bi-leveled factorization of $(k, k+1) w^{\prime}$ is

$$
u_{j}^{\prime} v^{1} u_{2}^{\prime} \ldots u_{j-1}^{\prime}\left(v^{j-1} u_{1}^{\prime} v^{j}\right) u_{j+1}^{\prime} \ldots u_{r}^{\prime} v^{r},
$$

and we necessarily have $\left(v^{1}, \ldots, v^{j-1} u_{1}^{\prime} v^{j}, \ldots, v^{r}\right) \leq \underline{\max }(c)$, which imples that $(k, k+1) w^{\prime} \leq \iota(c)$. We thus have the chain (2.3) in $\mathfrak{S}_{n}$, completing the proof.

If $b \lessdot c$ is the cover of type (iii) in Figure 6, the chain (2.3) from $\iota(b)$ to $\iota(c)$ is

$$
4357126 \leq 4367125 \lessdot 5367124 \leq 5467123 .
$$

\subsection{Tree enumeration. Let}

$$
\mathbf{S}(q):=\sum_{n \geq 0} n ! q^{n}=1+q+2 q^{2}+6 q^{3}+24 q^{4}+120 q^{5}+\cdots
$$

be the enumerating series of permutations, and define $\mathbf{M}(q)$ and $\mathbf{Y}(q)$ similarly

$$
\begin{aligned}
& \mathrm{M}(q):=\sum_{n \geq 0} A_{n} q^{n}=1+q+2 q^{2}+6 q^{3}+21 q^{4}+80 q^{5}+\cdots \\
& \mathrm{Y}(q):=\sum_{n \geq 0} C_{n} q^{n}=1+q+2 q^{2}+5 q^{3}+14 q^{4}+42 q^{5}+\cdots
\end{aligned}
$$

where $A_{n}:=\left|\mathcal{M}_{n}\right|$ and $C_{n}:=\left|\mathcal{Y}_{n}\right|$ are the Catalan numbers $\frac{1}{n+1}\left(\begin{array}{c}2 n \\ n\end{array}\right)$, whose enumerating series satisfies

$$
\mathbf{Y}(q)=\frac{1-\sqrt{1-4 q}}{2 q}=\frac{2}{1+\sqrt{1-4 q}} .
$$

Bi-leveled trees are Catalan-like [8, Theorem 3.1]: for $n \geq 1, A_{n}=C_{n-1}+$ $\sum_{i=1}^{n-1} A_{i} A_{n-i}$. See also [24, A121988]. Their enumerating series satisfies

$$
\mathbf{M}(q)=1+q \mathbf{Y}(q) \cdot \mathbf{Y}(q \mathbf{Y}(q)) \text {. }
$$

We will also be interested in $\mathbf{M}_{+}(q):=\sum_{n>0} A_{n} q^{n}=q \mathbf{Y}(q) \cdot \mathbf{Y}(q \mathbf{Y}(q))$.

Theorem 2.7. The only nontrivial quotients of the enumerating series $\mathbf{S}(q), \mathbf{M}(q)$, $\mathbf{M}_{+}(q)$, and $\mathbf{Y}(q)$ whose expansions have nonnegative coefficients are

$$
\mathbf{S}(q) / \mathbf{M}(q), \quad \mathbf{S}(q) / \mathbf{Y}(q), \quad \mathbf{M}_{+}(q) / \mathbf{Y}(q), \quad \text { and } \quad \mathbf{M}(q) / \mathbf{Y}(q)
$$


Proof. We prove the positivity of the quotient $\mathbf{S}(q) / \mathbf{M}(q)$ in Section 4.2. The positivity of $\mathbf{S}(q) / \mathbf{Y}(q)$ was established after [2, Theorem 7.2], which shows that $\mathfrak{S S y m}$ is a smash product over $\mathcal{Y}$ Sym.

For the positivity of $\mathbf{M}_{+}(q) / \mathbf{Y}(q)$, we use [3, Proposition 3], which computes $\mathbf{Y}(q \mathbf{Y}(q))=\sum_{n>0} B_{n} q^{n-1}$, where

$$
B_{1}:=C_{0} \quad \text { and } \quad B_{n}:=\sum_{k=0}^{n-1} \frac{k}{n-1}\left(\begin{array}{c}
2 n-k-3 \\
n-k-1
\end{array}\right) C_{k} \quad \text { for } n>1 .
$$

In particular, $B_{n} \geq 0$ for all $n \geq 0$. Returning to the quotient, we have

$$
\frac{\mathbf{M}_{+}(q)}{\mathbf{Y}(q)}=\frac{q \mathbf{Y}(q) \cdot \mathbf{Y}(q \mathbf{Y}(q))}{\mathbf{Y}(q)}=q \mathbf{Y}(q \mathbf{Y}(q)) \text {, }
$$

so $\mathbf{M}_{+}(q) / \mathbf{Y}(q)=\sum_{n>0} B_{n} q^{n}$ has nonnegative coefficients.

For $\mathbf{M}(q) / \mathbf{Y}(q)$, use the identity $1 / \mathbf{Y}(q)=1-q \mathbf{Y}(q)$ to obtain

$$
\frac{\mathbf{M}(q)}{\mathbf{Y}(q)}=\mathbf{M}_{+}(q)+1-q \mathbf{Y}(q)=1+\sum_{n>0}\left(B_{n}-C_{n-1}\right) q^{n} .
$$

Positivity is immediate as $B_{n}-C_{n-1} \geq 0$ for $n>0$.

We leave the proof that the remaining quotients have negative coefficients to the reader's computer.

Remark 2.8. Up to an index shift, the quotient $\mathbf{M}_{+}(q) / \mathbf{Y}(q)$ corresponds to the sequence [24, A127632] begining with $(1,1,3,11,44,185,804)$. We give a new combinatorial interpretation of this sequence in Corollary 4.3 .

\section{The Algebra MSym}

Let $\mathcal{M S y m}:=\bigoplus_{n \geq 0} \mathcal{M}$ Sym $_{n}$ denote the graded $\mathbb{Q}$-vector space whose $n^{\text {th }}$ graded piece has the basis $\left\{F_{b} \mid b \in \mathcal{M}_{n}\right\}$. The maps $\beta:$ S. $\rightarrow \mathcal{M}$. and $\phi: \mathcal{M}$. $\rightarrow$ $\mathcal{Y}$. of graded sets induce surjective maps of graded vector spaces

$$
\mathfrak{S} \text { Sym } \stackrel{\boldsymbol{\beta}}{\longrightarrow} \mathcal{M} \text { Sym } \stackrel{\phi}{\longrightarrow} \mathcal{Y} \text { Sym } \quad F_{w} \mapsto F_{\beta(w)} \mapsto F_{\phi(\beta(w))},
$$

which factor the Hopf algebra map $\boldsymbol{\tau}: \mathfrak{S S y m} \rightarrow \mathcal{Y}$ Sym, as $\phi(\beta(w))=\tau(w)$. We will show how the maps $\boldsymbol{\beta}$ and $\boldsymbol{\tau}$ induce on $\mathcal{M}$ Sym the structures of an algebra, of a $\mathfrak{S}$ Sym-module, and of a $\mathcal{Y} S y m$-comodule so that the composition (3.1) factors the map $\boldsymbol{\tau}$ as maps of algebras, of $\mathfrak{S} S y m$-modules, and of $\mathcal{Y} S y m$-comodules.

3.1. Algebra structure on $\mathcal{M}$ Sym. For $b, c \in \mathcal{M}$. define

$$
F_{b} \cdot F_{c}=\boldsymbol{\beta}\left(F_{w} \cdot F_{v}\right),
$$

where $w, v$ are permutations in $\mathbf{S}$. with $b=\beta(w)$ and $c=\beta(v)$.

Theorem 3.1. The operation $F_{b} \cdot F_{c}$ defined by (3.2) is independent of choices of $w, v$ with $\beta(w)=b$ and $\beta(v)=c$ and it endows $\mathcal{M}$ Sym with the structure of $a$ graded connected algebra such that the map $\boldsymbol{\beta}: \mathfrak{S} S y m \rightarrow \mathcal{M}$ Sym is a surjective map of graded connected algebras. 
If the expression $\boldsymbol{\beta}\left(F_{w} \cdot F_{v}\right)$ is independent of choice of $w \in \beta^{-1}(b)$ and $v \in \beta^{-1}(c)$, then the map $\boldsymbol{\beta}$ is automatically multiplicative. Associative and unital properties for $\mathcal{M}$ Sym are then inherited from those for $\mathfrak{S} S y m$, and the theorem follows. To prove independence (in Lemma 3.2), we formulate a description of (3.2) in terms of splittings and graftings of bi-leveled trees.

Let $s \stackrel{\curlyvee}{\rightarrow}\left(s_{0}, \ldots, s_{m}\right)$ be a splitting on the underlying tree of a bi-leveled tree $b=(s ; \mathrm{S}) \in \mathcal{M}_{n}$. Then the nodes of $s$ are distributed among the nodes of the partially ordered forest $\left(s_{0}, \ldots, s_{m}\right)$ so that the order ideal $\mathbf{S}$ gives a sequence of order ideals in the trees $s_{i}$. Write $b \stackrel{r}{\rightarrow}\left(b_{0}, \ldots, b_{m}\right)$ for the corresponding splitting of the bi-leveled tree $b$, viewing $b_{i}$ as $\left(s_{i} ;\left.\mathrm{S}\right|_{s_{i}}\right)$. (Note that only $b_{0}$ is guaranteed to be a bi-leveled tree.) Given $c=\left(t\right.$; T) $\in \mathcal{M}_{m}$ and a splitting $b \stackrel{r}{\rightarrow}\left(b_{0}, \ldots, b_{m}\right)$ of $b \in \mathcal{M}_{n}$, form a bi-leveled tree $\left(b_{0}, \ldots, b_{m}\right) / c$ whose underlying tree is $\left(s_{0}, \ldots, s_{m}\right) / t$ and whose order ideal is either

(i) $\mathrm{T}$, if $b_{0} \in \mathcal{M}_{0}$, or

(ii) $\mathrm{S} \cup\{$ the nodes of $t\}$, if $b_{0} \notin \mathcal{M}_{0}$.

Lemma 3.2. The product (3.2) is independent of choices of $w, v$ with $\beta(w)=b$ and $\beta(v)=c$. For $b \in \mathcal{M}_{n}$ and $c \in \mathcal{M}_{m}$, we have

$$
F_{b} \cdot F_{c}=\sum_{b \stackrel{\curlyvee}{\rightarrow}\left(b_{0}, \ldots, b_{m}\right)} F_{\left(b_{0}, \ldots, b_{m}\right) / c} .
$$

Proof. Fix any $w \in \beta^{-1}(b)$ and $v \in \beta^{-1}(c)$. The bi-leveled tree $\beta\left(\left(w_{0}, \ldots, w_{m}\right) / v\right)$ associated to a splitting $w \stackrel{r}{\rightarrow}\left(w_{0}, \ldots, w_{m}\right)$ has underlying tree $\left(s_{0}, \ldots, s_{m}\right) / t$, where $s \stackrel{r}{\rightarrow}\left(s_{0}, \ldots, s_{m}\right)$ is the induced splitting on the underlying tree $s=\tau(w)=$ $\phi(b)$. Each node of $\left(w_{0}, \ldots, w_{m}\right) / v$ comes from a node of either $w$ or $v$, with the labels of nodes from $w$ all smaller than the labels of nodes from $v$. Consequently, the leftmost node of $\left(w_{0}, \ldots, w_{m}\right) / v$ comes from either

(i) $v$, and then $\mathrm{T}\left(\left(w_{0}, \ldots, w_{m}\right) / v\right)=\mathrm{T}(v)=\mathrm{T}(c)$, or

(ii) $w$, and then $\mathrm{T}\left(\left(w_{0}, \ldots, w_{m}\right) / v\right)=\mathrm{T}(w)=\mathrm{T}(b) \cup\{$ the nodes of $v\}$.

The first case is when $w_{0} \in \mathfrak{S}_{0}$ and the second case is when $w_{0} \notin \mathfrak{S}_{0}$.

Here is the product $F_{y} \cdot F_{Y}$, together with the corresponding splittings of $\psi_{y}$

$$
\begin{aligned}
& F_{y} \cdot F_{y}=F_{y y}+F_{y}+F_{y y}+F_{y}+F_{y y}+F_{y} . \\
& \text { WII YH YM YY WY MY }
\end{aligned}
$$

3.2. SSym module structure on $\mathcal{M}$ Sym. Since $\boldsymbol{\beta}$ is a surjective algebra map, $\mathcal{M}$ Sym becomes a SSym-bimodule with the action

$$
F_{w} \cdot F_{b} \cdot F_{v}=F_{\beta(w)} \cdot F_{b} \cdot F_{\beta(v)} .
$$

The map $\boldsymbol{\tau}$ likewise induces on $\mathcal{Y} S y m$ the structure of a $\mathfrak{S} S y m$-bimodule, and the maps $\boldsymbol{\beta}, \boldsymbol{\phi}$, and $\boldsymbol{\tau}$ are maps of $\mathfrak{S} S y m$-bimodules. 
Curiously, we may use the map $\iota: \mathcal{M}$. $\rightarrow \mathfrak{S}$. to define the structure of a right $\mathfrak{s}$ Sym-comodule on $\mathcal{M}$ Sym,

$$
F_{b} \longmapsto \sum_{\iota(b) \stackrel{r}{\rightarrow}\left(w_{0}, w_{1}\right)} F_{\beta\left(w_{0}\right)} \otimes F_{w_{1}} .
$$

This induces a right comodule structure, because if $\iota(b) \stackrel{\curlyvee}{\rightarrow}\left(w_{0}, w_{1}\right)$, then $w_{0}=$ $\iota\left(\beta\left(w_{0}\right)\right)$, which may be checked using the characterization of $\iota$ in terms of pattern avoidance, as explained in Remark 2.3.

While $\mathcal{M}$ Sym is both a right $\mathfrak{S}$ Sym-module and right $\mathfrak{S} S y m$-comodule, it is not an $\mathfrak{S} S y m-$ Hopf module. For if it were a Hopf module, then the fundamental theorem of Hopf modules (see Remark 4.4) would imply that the series $\mathbf{M}(q) / \mathbf{S}(q)$ has positive coefficients, which contradicts Theorem 2.7.

3.3. Y Sym-comodule structure on $\mathcal{M} S y m$. For $b \in \mathcal{M}$., define the linear map $\boldsymbol{\rho}: \mathcal{M}$ Sym $\rightarrow \mathcal{M}$ Sym $\otimes \mathcal{Y}$ Sym by

$$
\boldsymbol{\rho}\left(F_{b}\right)=\sum_{\substack{\stackrel{\Upsilon}{\rightarrow}\left(b_{0}, b_{1}\right) \\ F_{b_{0}}}} \otimes F_{\phi\left(b_{1}\right)} .
$$

By $\phi\left(b_{1}\right)$, we mean the tree underlying $b_{1}$.

Example 3.3. In the fundamental bases of $\mathcal{M} S y m$ and $\mathcal{Y} S y m$, we have

$$
\boldsymbol{\rho}\left(F_{\gamma y}\right)=F_{\gamma y} \otimes 1+F_{Y} \otimes F_{Y}+F_{y} \otimes F_{Y}+F_{Y} \otimes F_{Y y}+1 \otimes F_{\curlyvee} y .
$$

Theorem 3.4. Under $\boldsymbol{\rho}, \mathcal{M} S y m$ is a right $\mathcal{Y}$ Sym-comodule.

Proof. This is counital as $(b, 1)$ is a splitting of $b$. Coassociativity is also clear as both $(\boldsymbol{\rho} \otimes 1) \boldsymbol{\rho}$ and $(1 \otimes \Delta) \boldsymbol{\rho}$ applied to $F_{b}$ for $b \in \mathcal{M}$. are sums of terms $F_{b_{0}} \otimes F_{\phi\left(b_{1}\right)} \otimes F_{\phi\left(b_{2}\right)}$ over all splittings $b \stackrel{r}{\rightarrow}\left(b_{0}, b_{1}, b_{2}\right)$.

Careful bookkeeping of the terms in $\boldsymbol{\rho}\left(F_{b} \cdot F_{c}\right)$ show that it equals $\boldsymbol{\rho}\left(F_{b}\right) \cdot \boldsymbol{\rho}\left(F_{c}\right)$ for all $b, c \in \mathcal{M}$. and thus $\mathcal{M}$ Sym is a $\mathcal{Y}$ Sym-comodule algebra. Hence, $\phi$ is a map of $\mathcal{Y} S y m-$ comodule algebras, and in fact $\boldsymbol{\beta}$ is also a map of $\mathcal{Y} S y m-$ comodule algebras. We leave this to the reader, and will not pursue it further.

Since $\boldsymbol{\tau}: \mathfrak{S} S y m \rightarrow \mathcal{Y}$ Sym is a map of Hopf algebras, $\mathfrak{S}$ Sym is naturally a right $\mathcal{Y}$ Sym-comodule where the comodule map is the composition

$$
\mathfrak{S} S y m \stackrel{\Delta}{\longrightarrow} \mathfrak{S} \text { Sym } \otimes \mathfrak{S} \text { Sym } \stackrel{1 \otimes \boldsymbol{\tau}}{\longrightarrow} \mathfrak{S} \text { Sym } \otimes \mathcal{Y} \text { Sym } .
$$

With these definitions, the following lemma is immedate.

Lemma 3.5. The maps $\boldsymbol{\tau}$ and $\boldsymbol{\phi}$ are maps of right $\mathcal{Y}$ Sym-comodules.

In particular, we have the equality of maps $\mathfrak{S} S y m \rightarrow \mathcal{M}$ Sym $\otimes \mathcal{Y} S y m$,

$$
\boldsymbol{\rho} \circ \boldsymbol{\beta}=(\boldsymbol{\beta} \otimes \boldsymbol{\tau}) \circ \Delta
$$


3.4. Coaction in the monomial basis. The coalgebra structures of $\mathfrak{S} S y m$ and $\mathcal{Y}$ Sym were elicudated by considering a second basis related to the fundamental basis via Möbius inversion. For $b \in \mathcal{M}_{n}$, define

$$
M_{b}:=\sum_{b \leq c} \mu(b, c) F_{c},
$$

where $\mu(\cdot, \cdot)$ is the Möbius function on the poset $\mathcal{M}_{n}$.

Given $b \in \mathcal{M}_{m}$ and $s \in \mathcal{Y}_{q}$, write $b \backslash s$ for the bi-leveled tree with $p+q$ nodes whose underlying tree is formed by grafting the root of $s$ onto the rightmost leaf of $b$, but whose order ideal is that of $b$. Here is an example of $b, s$, and $b \backslash s$,
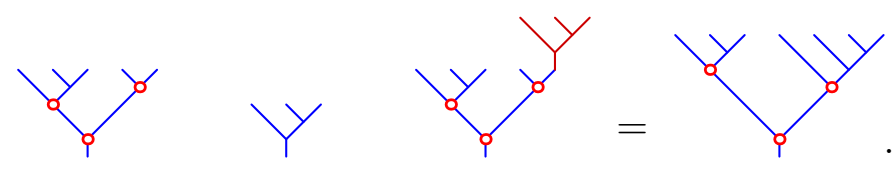

Observe that we cannot have $b=1$ in this construction.

The maximum bi-leveled tree with a given underlying tree $t$ is $\beta(\max (t))$, which has order ideal $\mathrm{T}$ consisting only of the nodes of $t$ along its leftmost branch. Here are three such trees of the form $\beta(\max (t))$,
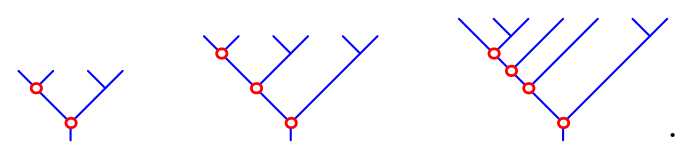

Theorem 3.6. Given $b=(t ; \mathrm{T}) \in \mathcal{M}$., we have

$$
\boldsymbol{\rho}\left(M_{b}\right)=\left\{\begin{array}{ll}
\sum_{b=c \backslash s} M_{c} \otimes M_{s} & \text { if } b \neq \beta(\max (t)) \\
\sum_{b=c \backslash s} M_{c} \otimes M_{s}+1 \otimes M_{t} & \text { if } b=\beta(\max (t))
\end{array} .\right.
$$

For example,

$$
\begin{aligned}
& \left.\rho\left(M_{\curlyvee}\right)=M_{\curlyvee}\right) \otimes 1 \\
& \boldsymbol{\rho}\left(M_{\gamma y}\right)=M_{\gamma y} \otimes 1+M_{\gamma} \otimes M_{Y}
\end{aligned}
$$

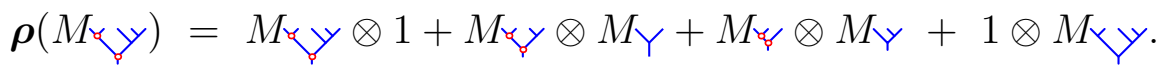

Our proof of Theorem 3.6 uses Proposition 1.3 and the following results.

Lemma 3.7. For any bi-leveled tree $b \in \mathcal{M}$., we have

$$
\boldsymbol{\beta}\left(\sum_{\beta(w)=b} M_{w}\right)=M_{b}
$$

Proof. Expand the left hand side in terms of the fundamental bases to get

$$
\boldsymbol{\beta}\left(\sum_{\beta(w)=b} \sum_{w \leq v} \mu_{\mathfrak{S}}(w, v) F_{v}\right)=\sum_{\beta(w)=b} \sum_{w \leq v} \mu_{\mathfrak{S}}(w, v) F_{\beta(v)} .
$$


As $\beta$ is surjective, we may change the index of summation to $b \leq c$ in $\mathcal{M}$. to obtain

$$
\sum_{b \leq c}\left(\sum_{\substack{\beta(w)=b \\ \beta(v)=c}} \mu_{\mathfrak{S}}(w, v)\right) F_{c} .
$$

By Theorems 1.4 and 2.4, the inner sum is $\mu_{\mathcal{M}}(b, c)$, so this sum is $M_{b}$.

Recall that $w=u \backslash v$ only if $\tau(w)=\tau(u) \backslash \tau(v)$ and the values of $w$ in the nodes of $u$ exceed the values in the nodes of $v$. We always have the trivial decomposition $w=(\emptyset, w)$. Suppose that $w=u \backslash v$ with $u \neq \emptyset$ a nontrivial decomposition. If $\beta(w)=b=(t ; \mathrm{T})$, then $\mathrm{T}$ is a subset of the nodes of $u$ so that $\beta(u)=(\tau(u) ; \mathrm{S})$ and $b=\beta(u) \backslash \tau(v)$. Moreover, for every decomposition $b=c \backslash s$ and every $u, v$ with $\beta(u)=c$ and $\tau(v)=s$, we have $b=\beta(u \backslash v)$. Thus, for $b \in \mathcal{M}$., we have

$$
\bigsqcup_{\beta(w)=b} \bigsqcup_{\begin{array}{c}
w=u \backslash v \\
u \neq \emptyset
\end{array}}(u, v)=\bigsqcup_{b=c \backslash t} \bigsqcup_{\beta(u)=c} \bigsqcup_{\tau(v)=t}(u, v) .
$$

Proof of Theorem 3.6. Let $b=(t$; T) with $t \neq 1$. Using Lemma 3.7, we have

$$
\boldsymbol{\rho}\left(M_{b}\right)=\boldsymbol{\rho} \boldsymbol{\beta}\left(\sum_{\beta(w)=b} M_{w}\right)=\sum_{\beta(w)=b} \boldsymbol{\rho} \boldsymbol{\beta} M_{w} .
$$

By (3.5), (3.7), and (1.5), this equals

$$
\begin{aligned}
\sum_{\beta(w)=b} \sum_{\substack{w=u \backslash v \\
u \neq \emptyset}} \boldsymbol{\beta}\left(M_{u}\right) \otimes \boldsymbol{\tau}\left(M_{v}\right)+\sum_{\beta(w)=b} \boldsymbol{\beta}\left(M_{\emptyset}\right) \otimes \boldsymbol{\tau}\left(M_{w}\right) \\
\quad=\sum_{b=c \backslash s}\left(\sum_{\beta(u)=c} \boldsymbol{\beta}\left(M_{u}\right)\right) \otimes\left(\sum_{\tau(v)=s} \boldsymbol{\tau}\left(M_{t}\right)\right)+\sum_{\beta(w)=b} 1 \otimes \boldsymbol{\tau}\left(M_{w}\right) .
\end{aligned}
$$

By Lemma 3.7 and (1.4), the first sum becomes $\sum_{b=c \backslash s} M_{c} \otimes M_{s}$ and the second sum vanishes unless $b=\beta(\max (t))$. This completes the proof.

\section{Hopf VARiations}

4.1. The $\mathcal{Y} S y m-$ Hopf module $\mathcal{M} S y m_{+}$. Let $\mathcal{M}_{+}:=\left(\mathcal{M}_{n}\right)_{n \geq 1}$ be the bi-leveled trees with at least one internal node and define $\mathcal{M} S y m_{+}$to be the positively graded part of $\mathcal{M S y m}$, which has bases indexed by $\mathcal{M}_{+}$. A restricted splitting of $b \in \mathcal{M}_{+}$is a splitting $b \stackrel{\Upsilon_{+}}{\longrightarrow}\left(b_{0}, \ldots, b_{m}\right)$ with $b_{0} \in \mathcal{M}_{+}$, i.e., $b_{0} \neq 1$. Given $b \stackrel{\Upsilon_{+}}{\longrightarrow}\left(b_{0}, \ldots, b_{m}\right)$ and $t \in \mathcal{Y}_{m}$, form the bi-leveled tree $\left(b_{0}, \ldots, b_{m}\right) / t$ by grafting the ordered forest $\left(b_{0}, \ldots, b_{m}\right)$ onto the leaves of $t$, with order ideal consisting of the nodes of $t$ together with the nodes of the forest coming from the order ideal of $b$, as in $(3.3)(i i)$.

We define an action and coaction of $\mathcal{Y} S y m$ on $\mathcal{M S y m}$ that are similar to the product and coaction on $\mathcal{M S y m}$. They come from a second collection of polytope 
maps $\mathcal{M}_{n} \rightarrow \mathcal{Y}_{n-1}$ arising from viewing the vertices of $\mathcal{M}_{n}$ as painted trees on $n-1$ nodes (see $[5,8])$. For $b \in \mathcal{M}_{+}$and $t \in \mathcal{Y}_{m}$, set

$$
\begin{aligned}
& F_{b} \cdot F_{t}=\sum_{b \stackrel{\Upsilon_{+}}{\longrightarrow}\left(b_{0}, \ldots, b_{m}\right)} F_{\left(b_{0}, \ldots, b_{m}\right) / t}, \\
& \rho_{+}\left(F_{b}\right)=\sum_{b \stackrel{\Upsilon_{+}}{\longrightarrow}\left(b_{0}, b_{1}\right)} F_{b_{0}} \otimes F_{\phi\left(b_{1}\right)} .
\end{aligned}
$$

For example, in the fundamental bases of $\mathcal{M} S y m_{+}$and $\mathcal{Y} S y m$, we have

$$
\begin{aligned}
F_{Y} \cdot F_{Y} & =F_{Y y}+F_{Y}+F_{y y}, \\
\boldsymbol{\rho}_{+}\left(F_{Y y}\right) & =F_{Y y} \otimes 1+F_{Y} \otimes F_{Y}+F_{Y} \otimes F_{Y}+F_{Y} \otimes F_{\nvdash y} .
\end{aligned}
$$

Theorem 4.1. The operations in (4.1) define a Y Sym-Hopf module structure on $\mathcal{M S y m}_{+}$.

Proof. The unital and counital properties are immediate. We check only that the action is associative, the coaction is coassociative, and the two structures commute with each other.

Associativity. Fix $b=(t ; \mathrm{T}) \in \mathcal{M}_{+}, r \in \mathcal{Y}_{m}$, and $s \in \mathcal{Y}_{n}$. A term in the expression $\left(F_{b} \cdot F_{r}\right) \cdot F_{s}$ corresponds to a restricted splitting and grafting $b \stackrel{\Upsilon_{+}}{\longrightarrow}$ $\left(b_{0}, \ldots, b_{m}\right) \rightsquigarrow\left(b_{0}, \ldots, b_{m}\right) / r=c$, followed by another $c \stackrel{\Upsilon_{+}}{\longrightarrow}\left(c_{0}, \ldots, c_{n}\right) \rightsquigarrow$ $\left(c_{0}, \ldots, c_{n}\right) / t$. The order ideal for this term equals $\mathrm{T} \cup\{$ the nodes of $r$ and $s\}$. Note that restricted splittings of $c$ are in bijection with pairs of splittings

$$
\left(b \stackrel{\Upsilon_{+}}{\longrightarrow}\left(b_{0}, \ldots, b_{m+n}\right), r \stackrel{\Upsilon}{\rightarrow}\left(r_{0}, \ldots, r_{n}\right)\right) .
$$

Terms of $F_{b} \cdot\left(F_{r} \cdot F_{s}\right)$ also correspond to these pairs of splittings. The order ideal for this term is again $\mathrm{T} \cup\{$ the nodes of $r$ and $s\}$. That is, $\left(F_{b} \cdot F_{r}\right) \cdot F_{s}$ and $F_{b} \cdot\left(F_{r} \cdot F_{s}\right)$ agree term by term.

Coassociativity. Fix $b=(t ; \mathrm{T}) \in \mathcal{M}_{+}$. Terms $F_{c} \otimes F_{r} \otimes F_{s}$ in $\left(\boldsymbol{\rho}_{+} \otimes \mathbb{1}\right) \boldsymbol{\rho}_{+}\left(F_{b}\right)$ and $(\mathbb{1} \otimes \Delta) \boldsymbol{\rho}_{+}\left(F_{b}\right)$ both correspond to restricted splittings $b \stackrel{\Upsilon_{+}}{\longrightarrow}\left(c, c_{1}, c_{2}\right)$, where $\phi\left(c_{1}\right)=r$ and $\phi\left(c_{2}\right)=s$. In either case, the order ideal on $c$ is $\left.\mathrm{T}\right|_{c}$.

Commuting structures. Fix $b=(s ; \mathrm{S}) \in \mathcal{M}_{+}$and $t \in \mathcal{Y}_{m}$. A term $F_{c_{0}} \otimes F_{\phi\left(c_{1}\right)}$ in $\boldsymbol{\rho}_{+}\left(F_{b} \cdot F_{t}\right)$ corresponds to a choice of a restricted splitting and grafting $b \stackrel{\Upsilon_{+}}{\longrightarrow}$ $\left(b_{0}, \ldots, b_{m}\right) \rightsquigarrow\left(b_{0}, \ldots, b_{r}\right) / t=c$, followed by a restricted splitting $c \stackrel{\Upsilon_{+}}{\longrightarrow}\left(c_{0}, c_{1}\right)$. The order ideal on $c_{0}$ equals the nodes of $c_{0}$ inherited from $\mathrm{S}$, together with the nodes of $c_{0}$ inherited from $t$. The restricted splittings of $c$ are in bijection with pairs of splittings $\left(b \stackrel{\Upsilon_{+}}{\longrightarrow}\left(b_{0}, \ldots, b_{m+1}\right), t \stackrel{\curlyvee}{\longrightarrow}\left(t_{0}, t_{1}\right)\right)$. If $t_{0} \in \mathcal{Y}_{n}$, then the pair of graftings $c_{0}=\left(b_{0}, \ldots, b_{n}\right) / t_{0}$ and $c_{1}=\left(b_{n+1}, \ldots, b_{m}\right) / t_{1}$ are precisely the terms appearing in $\boldsymbol{\rho}_{+}\left(F_{b}\right) \cdot \Delta\left(F_{t}\right)$.

The similarity of (4.1) to the coaction (3.4) of $\mathcal{Y}$ Sym on $\mathcal{M}$ Sym gives the following result, whose proof we leave to the reader. 
Corollary 4.2. For $b \in \mathcal{M}_{+}$, we have

$$
\boldsymbol{\rho}_{+}\left(M_{b}\right)=\sum_{b=c \backslash s} M_{c} \otimes M_{s} .
$$

This elucidates the structure of $\mathcal{M}$ Sym $_{+}$. Let $\mathcal{B} \subset \mathcal{M}_{+}$be the indecomposable bi-leveled trees - those with only trivial decompositions, $b=b \backslash$. Then $(t ; \mathrm{T}) \in \mathcal{B}$ if and only if $\mathrm{T}$ contains the rightmost node of $t$. Every tree $c$ in $\mathcal{M}_{+}$has a unique

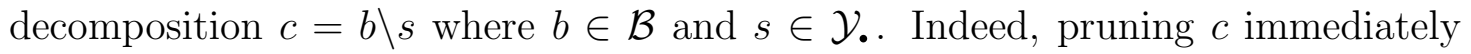
above the rightmost node in its order ideal gives a decomposition $c=b \backslash s$ where $b \in \mathcal{B}$ and $s \in \mathcal{Y}_{.}$. This induces a bijection of graded sets,

$$
\mathcal{M}_{+} \longleftrightarrow \mathcal{B} \times \mathcal{Y}_{.} .
$$

Moreover, if $b \in \mathcal{B}$ and $s \in \mathcal{Y}_{\bullet}$, then Corollary 4.2 and (1.6) together imply that

$$
\boldsymbol{\rho}_{+}\left(M_{b \backslash s}\right)=\sum_{s=r \backslash t} M_{b \backslash r} \otimes M_{t} .
$$

Note that $\mathbb{Q} \mathcal{B} \otimes \mathcal{Y} S y m$ is a graded right $\mathcal{Y} S y m$-comodule with structure map,

$$
b \otimes M_{s} \longmapsto b \otimes\left(\Delta M_{s}\right)
$$

for $b \in \mathcal{B}$ and $s \in \mathcal{Y}_{.}$. Comparing this with (4.2), we deduce the following algebraic and combinatorial facts.

Corollary 4.3. The map $\mathbb{Q B} \otimes \mathcal{Y} S y m \rightarrow \mathcal{M} S y m_{+}$defined by $b \otimes M_{s} \mapsto M_{b \backslash s}$ is an isomorphism of graded right $\mathcal{Y} S y m$ comodules.

The quotient of enumerating series $\mathbf{M}(q)_{+} / \mathbf{Y}(q)$ is equal to the enumerating series of the graded set $\mathcal{B}$.

In particular, if $\mathcal{B}_{n}:=\mathcal{B} \cap \mathcal{M}_{n}$, then $\left|\mathcal{B}_{n}\right|=B_{n}$ by $(2.5)$.

Remark 4.4. The coinvariants in a right comodule $M$ over a coalgebra $C$ are $M^{\text {co }}:=\{m \in M \mid \boldsymbol{\rho}(m)=m \otimes 1\}$. We identify the vector space $\mathbb{Q} \mathcal{B}$ with $\mathcal{M} S y m_{+}^{\text {co }}$ via $b \mapsto M_{b}$. The isomorphism $\mathbb{Q B} \otimes \mathcal{Y} S y m \rightarrow \mathcal{M}$ Sym $_{+}$is a special case of the Fundamental Theorem of Hopf Modules [19, Theorem 1.9.4]: If $M$ is a Hopf module over a Hopf algebra $H$, then $M \simeq M^{\text {co }} \otimes H$ as Hopf modules.

4.2. Hopf module structure on MSym. We use Theorem 3.6 to identify the $\mathcal{Y}$ Sym-coinvariants in $\mathcal{M}$ Sym. Let $\mathcal{B}^{\prime}$ be those indecomposable bi-leveled trees which are not of the form $\beta(\max (t))$, for some $t \in \mathcal{Y}_{+}$, together with $\{\mid\}$.

Corollary 4.5. The $\mathcal{Y} S y m$-coinvariants of $\mathcal{M}$ Sym have a basis $\left\{M_{b} \mid b \in \mathcal{B}^{\prime}\right\}$.

For $n>0$, the difference $\mathcal{B}_{n} \backslash \mathcal{B}_{n}^{\prime}$ consists of indecomposable bi-leveled trees with $n$ nodes of the form $\beta(\max (t))$. If $\beta(\max (t)) \in \mathcal{B}_{n}$, then $t=s \vee \mathrm{I}$, for some $s \in \mathcal{Y}_{n-1}$, and so $\left|\mathcal{B}_{n}^{\prime}\right|=B_{n}-C_{n-1}$, which we saw in the proof of Theorem 2.7.

For $t \in \mathcal{Y}_{\text {. }}$, set $\mid \backslash t:=\beta(\max (t))$, and if $\mid \neq b \in \mathcal{B}^{\prime}$, set $b \rrbracket t:=b \backslash t$. Every bi-

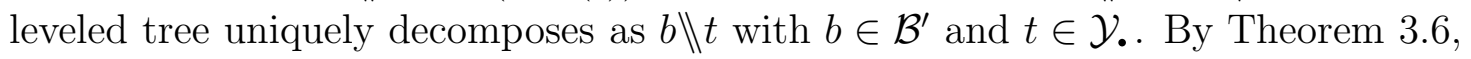
$M_{b} \otimes M_{t} \mapsto M_{b \Downarrow t}$ induces an isomorphism of right $\mathcal{Y} S y m$-comodules,

$$
\mathcal{M S y m}{ }^{\mathrm{co}} \otimes \mathcal{Y} \text { Sym } \longrightarrow \mathcal{M S y m},
$$


where the structure map on $\mathcal{M} S y m^{\mathrm{co}} \otimes \mathcal{Y} S y m$ is $M_{b} \otimes M_{t} \mapsto M_{b} \otimes \Delta\left(M_{t}\right)$. Treating $\mathcal{M S y m}^{\mathrm{co}}$ as a trivial $\mathcal{Y} S y m$-module, $M_{b} \cdot M_{t}=\varepsilon\left(M_{t}\right) M_{b}, \mathcal{M} S y m^{\mathrm{co}} \otimes \mathcal{Y} S y m$ becomes a right $\mathcal{Y} S y m$-module. As explained in [19, Example 1.9.3], this makes $\mathcal{M}$ Sym $^{\mathrm{co}} \otimes \mathcal{Y}$ Sym into a $\mathcal{Y}$ Sym-Hopf module.

We express this structure on $\mathcal{M}$ Sym. Let $b \rrbracket t \in \mathcal{M}$. and $s \in \mathcal{Y}$., then

$$
M_{b \Downarrow t} \cdot M_{s}=\sum_{r \in t \cdot s} M_{b \Downarrow r} \quad \text { and } \quad \rho\left(M_{b \Downarrow t}\right)=\sum_{t=r \backslash s} M_{b \Downarrow r} \otimes M_{s},
$$

where $t \cdot s$ is the set of trees $r$ indexing the product $M_{t} \cdot M_{s}$ in $\mathcal{Y} S y m$. The coaction is as before, but the product is new. It is not positive in the fundamental basis,

$$
F_{Y} \cdot F_{Y}=F_{Y}-F_{Y}+F_{Y}+2 F_{Y y} \text {. }
$$

We complete the proof of Theorem 2.7.

Corollary 4.6. The power series $\mathbf{S}(q) / \mathbf{M}(q)$ has nonnegative coefficients.

Proof. Observe that

$$
\mathbf{S}(q) / \mathbf{M}(q)=(\mathbf{S}(q) / \mathbf{Y}(q)) /(\mathbf{M}(q) / \mathbf{Y}(q))
$$

Since both SSym and $\mathcal{M}$ Sym are right $\mathcal{Y}$ Sym-Hopf modules, the two quotients of enumerating series on the right are generating series for their coinvariants, by the Fundamental Theorem of Hopf modules. Thus

$$
\mathbf{S}(q) / \mathbf{M}(q)=\mathbf{S}^{\mathrm{co}}(q) / \mathbf{M}^{\mathrm{co}}(q),
$$

where $\mathbf{S}^{\mathrm{co}}(q)$ and $\mathbf{M}^{\mathrm{co}}(q)$ are the enumerating series for $\mathfrak{S} S y m^{\mathrm{co}}$ and $\mathcal{M}$ Sym $\mathbf{m}^{\mathrm{co}}$. To show that $\mathbf{S}^{\mathrm{co}}(q) / \mathbf{M}^{\mathrm{co}}(q)$ is nonnegative, we index bases for these spaces by graded sets $\mathcal{S}$ and $\mathcal{B}^{\prime}$, then establish a bijection $\mathcal{B}^{\prime} \times \mathcal{S}^{\prime} \rightarrow \mathcal{S}$ for some graded subset $\mathcal{S}^{\prime} \subset \mathcal{S}$.

The set $\mathcal{B}^{\prime}$ was identified in Corollary 4.5. The coinvariants $\mathfrak{S} S y m^{\text {co }}$ were given in [2, Theorem 7.2] as a left Hopf kernel. The basis was identified as follows. Recall that pemutations $u \in \mathfrak{S}$. may be written uniquely in terms of indecomposables,

$$
u=u_{1} \backslash \cdots \backslash u_{r}
$$

(taking $r=0$ for $u=\emptyset$ ). Let $\mathcal{S} \subset \mathfrak{S}$. be those permutations $u$ whose rightmost indecomposable component has a 132-pattern, and thus $u \neq \max (t)$ for any $t \in \mathcal{Y}_{+}$. (Note that $u=\emptyset \in \mathcal{S}$.) Then $\left\{M_{u} \mid u \in \mathcal{S}\right\}$ is a basis for $\mathfrak{S} \operatorname{Sym}^{\text {co }}$.

Fix a section $g: \mathcal{M}$. $\rightarrow \mathfrak{S}$. of the map $\beta: \mathfrak{S}$. $\rightarrow \mathcal{M}$. and define a subset $\mathcal{S}^{\prime} \subset \mathcal{S}$ as follows. Given the decomposition $u=u_{1} \backslash \cdots \backslash u_{r}$ in (4.5) with $r \geq 0$, consider the length $\ell \geq 0$ of the maximum initial sequence $u_{1} \backslash \cdots \backslash u_{\ell}$ of indecomposables belonging to $g\left(\mathcal{B}^{\prime}\right)$. Put $u \in \mathcal{S}^{\prime}$ if $\ell$ is even. Define the map of graded sets

$$
\kappa: \mathcal{B}^{\prime} \times \mathcal{S}^{\prime} \longrightarrow \mathcal{S} \quad \text { by } \quad(b, v) \longmapsto g(b) \backslash v .
$$

The image of $\kappa$ lies in $\mathcal{S}$ as the last component of a nontrivial $g(b) \backslash v$ is either $g(b)$ or the last component of $v$, neither of which can be $\max (t)$ for $t \in \mathcal{Y}_{+}$.

We claim that $\kappa$ is bijective. If $u \in \mathcal{S}^{\prime}$, then $u=\kappa(\mid, u)$. If $u \in \mathcal{S} \backslash \mathcal{S}^{\prime}$, then $u$ has an odd number of initial components from $g\left(\mathcal{B}^{\prime}\right)$. Letting its first factor be $g(b)$, 
we see that $u=g(b) \backslash u^{\prime}=\kappa\left(b, u^{\prime}\right)$ with $u^{\prime} \in \mathcal{S}^{\prime}$. This surjective map is injective as the expressions $\kappa\left(\mathrm{I}, u^{\prime}\right)$ and $\kappa\left(b, u^{\prime}\right)$ with $b \in \mathcal{B}_{+}^{\prime}$ and $u^{\prime} \in \mathcal{S}^{\prime}$ are unique.

This isomorphism of graded sets identifies the enumerating series of the graded set $\mathcal{S}^{\prime}$ as the quotient $\mathbf{S}^{\mathrm{co}}(q) / \mathbf{M}^{\mathrm{co}}(q)$, which completes the proof.

\section{REFERENCES}

1. Marcelo Aguiar and Frank Sottile, Structure of the Malvenuto-Reutenauer Hopf algebra of permutations, Adv. Math. 191 (2005), no. 2, 225-275.

2. _ Structure of the Loday-Ronco Hopf algebra of trees, J. Algebra 295 (2006), no. 2, 473-511.

3. Paul Barry, A Catalan transform and related transformations on integer sequences, J. Integer Seq. 8 (2005), no. 4, Article 05.4.5, 24 pp. (electronic).

4. Anders Björner and Michelle L. Wachs, Shellable nonpure complexes and posets. II, Trans. Amer. Math. Soc. 349 (1997), no. 10, 3945-3975.

5. J. M. Boardman and R. M. Vogt, Homotopy invariant algebraic structures on topological spaces, Lecture Notes in Mathematics, Vol. 347, Springer-Verlag, Berlin, 1973.

6. Frédéric Chapoton, Algèbres de Hopf des permutahèdres, associahèdres et hypercubes, Adv. Math. 150 (2000), no. 2, 264-275.

7. Sergey Fomin and Andrei Zelevinsky, $Y$-systems and generalized associahedra, Ann. of Math. (2) 158 (2003), no. 3, 977-1018.

8. Stefan Forcey, Convex hull realizations of the multiplihedra, Topology Appl. 156 (2008), no. 2, $326-347$.

9. Stefan Forcey, Aaron Lauve, and Frank Sottile, Constructing cofree combinatorial coalgebras, in preparation.

10. Georges-Théodule Guilbaud and Pierre Rosenstiehl, Analyse algébrique d'un scrutin, Math. Sci. Humaines (1963), no. 4, 9-33.

11. Mark Haiman, Constructing the associahedron, unpublished manuscript, MIT, 1984.

12. Norio Iwase and Mamoru Mimura, Higher homotopy associativity, Algebraic topology (Arcata, CA, 1986), Lecture Notes in Math., vol. 1370, Springer, Berlin, 1989, pp. 193-220.

13. Carl W. Lee, The associahedron and triangulations of the n-gon, European J. Combin. 10 (1989), no. 6, 551-560.

14. Jean-Louis Loday and María O. Ronco, Hopf algebra of the planar binary trees, Adv. Math. 139 (1998), no. 2, 293-309.

15. _ Order structure on the algebra of permutations and of planar binary trees, J. Algebraic Combin. 15 (2002), no. 3, 253-270.

16. Claudia Malvenuto and Christophe Reutenauer, Duality between quasi-symmetric functions and the Solomon descent algebra, J. Algebra 177 (1995), no. 3, 967-982.

17. R. James Milgram, Iterated loop spaces, Ann. of Math. (2) 84 (1966), 386-403.

18. John W. Milnor, folklore: unpublished and unrecorded.

19. Susan Montgomery, Hopf algebras and their actions on rings, CBMS Regional Conference Series in Mathematics, vol. 82, Published for the Conference Board of the Mathematical Sciences, Washington, DC, 1993.

20. Nathan Reading, Lattice congruences of the weak order, Order 21 (2004), no. 4, 315-344 (2005).

21. Gian-Carlo Rota, On the foundations of combinatorial theory. I. Theory of Möbius functions, Z. Wahrscheinlichkeitstheorie und Verw. Gebiete 2 (1964), 340-368 (1964), Reprinted in: J.P.S. Kunt (Ed.), Gian-Carlo Rota on Combinatorics: Introductory Papers and Commentaires, Birkhäuser, Boston, 1995.

22. Samson Saneblidze and Ronald Umble, Diagonals on the permutahedra, multiplihedra and associahedra, Homology Homotopy Appl. 6 (2004), no. 1, 363-411 (electronic). 
23. Pieter Hendrik Schoute, Analytic treatment of the polytopes regularly derived from the regular polytopes, Verhandelingen der Koninklijke Akademie van Wetenschappen te Amsterdam. Eerste Sectie 11 (1911), no. 3, 1-87.

24. N. J. A. Sloane, The on-line encyclopedia of integer sequences, published electronically at www.research. att. com/ njas/sequences/.

25. James Stasheff, Homotopy associativity of H-spaces. I, II, Trans. Amer. Math. Soc. 108 (1963), 275-292; ibid. 108 (1963), 293-312.

26. _ H-spaces from a homotopy point of view, Lecture Notes in Mathematics, Vol. 161, Springer-Verlag, Berlin, 1970.

27. Andy Tonks, Relating the associahedron and the permutohedron, Operads: Proceedings of Renaissance Conferences (Hartford, CT/Luminy, 1995), Contemp. Math., vol. 202, Amer. Math. Soc., Providence, RI, 1997, pp. 33-36.

(S. Forcey) Department of Mathematics, Tennessee State University, 3500 John A Merritt Blvd, Nashyille, Tennessee 37209

E-mail address: sforcey@tnstate.edu

$U R L:$ http://faculty.tnstate.edu/sforcey/

(A. Lauve) Department of Mathematics, Texas A\&M University, MS 3368, ColLege Station, Texas 77843

E-mail address: lauve@math.tamu.edu

URL: http://www. math.tamu.edu/ lauve

(F. Sottile) Department of Mathematics, Texas A\&M University, MS 3368, ColLEge Station, Texas 77843

E-mail address: sottile@math.tamu.edu

$U R L:$ http://www.math.tamu.edu/ ${ }^{\text {sottile }}$ 